\title{
A new population of cool stars and brown dwarfs in the Lupus clouds ${ }^{\star, \star \star}$
}

\author{
F. Comerón ${ }^{1, \star \star \star}$, L. Spezzi ${ }^{2,3}$, and B. López Martí ${ }^{4}$ \\ ${ }^{1}$ ESO, Karl-Schwarzschild-Strasse 2, 85748 Garching, Germany \\ e-mail: fcomeron@eso.org \\ 2 INAF - Osservatorio Astrofisico di Catania, via S. Sofia, 78, 95123 Catania, Italy \\ 3 Research and Scientific Support Department, European Space Agency (ESTEC), PO Box 299, 2200 AG Noordwijk, \\ The Netherlands \\ ${ }^{4}$ Laboratorio de Astrofísica Estelar y Exoplanetas - Centro de Astrobiología (LAEX-CAB/INTA-CSIC, LAEFF-Apdo. 78, \\ 28691 Villanueva de la Cañada, Spain
}

Received 2 February 2009 / Accepted 6 April 2009

\section{ABSTRACT}

\begin{abstract}
Context. Most studies of the stellar and substellar populations of star-forming regions rely on using the signatures of accretion, outflows, disks, or activity characterizing the early stages of stellar evolution. However, these signatures rapidly decay with time. Aims. We present the results of a wide-area study of the stellar population of clouds in the Lupus star-forming region. When combined with 2MASS photometry, our data allow us to fit the spectral energy distributions of over 150000 sources and identify possible new members based on their photospheric fluxes, independent of any display of the signposts of youth.

Methods. We used the Wide Field Imager (WFI) at the La Silla $2.2 \mathrm{~m}$ telescope to image an area of more than 6 square degrees in the Lupus 1, 3 and 4 clouds in the $R_{\mathrm{C}}, I_{\mathrm{C}}$, and $z_{\mathrm{WFI}}$ bands, selected so as to overlap with the areas observed in the Spitzer Legacy Program "From molecular cores to planet-forming disks". We complement our data with 2MASS photometry to sample the spectral energy distribution from $0.6 \mu \mathrm{m}$ to $2.2 \mu \mathrm{m}$. We validate our method on the census of known members of the Lupus clouds, for which spectroscopic classification is available. The temperatures derived for cool objects are generally accurate, with most of the exceptions attributed to veiling, strong emission lines at short wavelengths, near-infrared excess, variability, or the presence of close companions. Results. Considering that the dereddened fluxes of most cool $\left(T_{\text {eff }}<3500 \mathrm{~K}\right)$ young stellar objects at the distance of Lupus occupy a gap between those typical both of field cool dwarfs and of background giants, we identify a new population of cool members of Lupus 1 and 3. The approximately 130 new members are only moderately concentrated toward the densest clouds, they appear to have ages in the same range as the known members, and very few show the infrared excess caused by warm disks. This population is absent in Lupus 4.

Conclusions. This new population of Lupus members seems to be composed of stars and brown dwarfs that have lost their inner disks on a timescale of a few Myr or less. Almost all these objects are in low extinction regions. We speculate that dissipation of unshielded disks caused by nearby $\mathrm{O}$ stars or fast collapse of the pre-(sub)stellar cores triggered by the passage of old supernova shocks may have led to disk properties and evolutionary paths very different from those resulting from the more quiescent environment provided by dense molecular clouds.
\end{abstract}

Key words. stars: low-mass, brown dwarfs - stars: formation - stars: pre-main sequence - ISM: individual objects: Lupus - ISM: clouds

\section{Introduction}

Much of our current knowledge of the earliest stages of the evolution of very low mass stars and brown dwarfs, the signatures of stellar and substellar youth, and the build-up and shape of the initial mass function comes from the observation of a number of star-forming regions within 150-200 pc from the Sun (see Reipurth 2008 for an extensive collection of reviews). The availability of instruments with large format, sensitive detectors operating at different wavelengths both on the ground and in space has made it possible to obtain an increasingly complete census of the young populations in all these regions down to masses

\footnotetext{
* Based on observations carried out at the ESO/La Silla, Chile under proposals numbers 69.C-0260(A) and 71.C-0259(A).

$\star \star$ Tables 4 and 9 are only available in electronic form at

http://www . aanda.org

$\star \star \star$ Visiting scientist at the Vatican Observatory.
}

well below the substellar limit. The approach normally adopted consists of using different telltale features displayed by young stellar objects as a way to identify members, and derive their intrinsic properties by means of detailed follow-up observations or with the help of photometric information.

The Lupus clouds, a complex of low-mass star-forming regions in the Scorpius-Centaurus OB association, has been the target of a number of such investigations (see Comerón 2008 for a historical review and a summary of existing results). Those clouds are spread over a large area of the sky, nearly $20^{\circ}$ across, with galactic longitudes in the range $334^{\circ}<l<352^{\circ}$ and latitudes $5^{\circ}<b<25^{\circ}$. Following early slitless spectroscopy surveys by Thé (1962) and Schwartz (1977) which revealed for the first time the rich T Tauri content of the Lupus clouds, their lowest mass component has been probed by more recent studies at different wavelengths. Hughes et al. (1994) obtained spectroscopy and infrared photometry for the members listed 
by Schwartz (1977). The Lupus 3 cloud, which contains the largest number and density of members, has been the target of several surveys: Nakajima et al. (2000) identified some possible very low luminosity members by means of near-infrared excesses. Comerón et al. (2003) added four new members identified through their $\mathrm{H} \alpha$ emission, including a M 8 brown dwarf. López Martí et al. (2005) have proposed 22 additional candidate members of Lupus 3 based on a wide-area survey using a combination of filters that allowed them to identify possible $\mathrm{H} \alpha$-emitting members and to estimate their temperatures from intermediate-band differential photometry near selected spectral features. Deep X-ray observations of Lupus 3, sensitive to the emission from brown dwarfs, have been presented by Gondoin (2006).

The most comprehensive recent searches for members of the Lupus clouds have been based on the results obtained by the Spitzer Space Observatory. As a result of the Spitzer Legacy program From molecular cores to planet-forming disks (Evans et al. 2003, 2008), a number of papers (Allers et al. 2006; Chapman et al. 2007; Merín et al. 2008) have reported on the discovery of new low-mass members and the confirmation of previously suspected ones. Further Spitzer observations of the region have been presented by Allen et al. (2007). These identifications are based on the determination of spectral energy distributions at infrared wavelengths, where warm dust dominates the emission. Merín et al. (2008) have synthesized the Spitzer findings on the Lupus 1, 3, and 4 clouds, and have discussed the properties of both the central objects and their circumstellar material. Together with the results of previous surveys, the member lists presented by Merín et al. (2008) represent the most complete census to date of stars and brown dwarfs in Lupus 1, 3, and 4.

Despite their wide coverage and the ability to reveal very low mass members, surveys based on the detection of $\mathrm{H} \alpha$ emission or infrared excesses caused by warm dust leave the question open about the fraction of members of a star-forming region that may not be accreting or surrounded by a sufficient amount of dust. Observational evidence points toward disk lifetimes of only a few Myr, with a significant fraction of stars losing their disks over a timescale that is comparable to the duration of the embedded phase (e.g. Haisch et al. 2001). In fact, as pointed out by Merín et al. (2008), some confirmed members of the star-forming region observed by Spitzer do not meet the infrared excess-based color criteria for their selection of candidates. Unbiased surveys where the identification of members is based solely on the photospheric properties (e.g. Luhman 2007) appear thus as a necessary complement of those based on the identification of signatures of accretion, dust, or the strong chromospheric activity characteristic of young stellar objects.

In this paper we present the results of a broad-band, widearea visible imaging survey of the Lupus 1, 3, and 4 clouds complemented with near-infrared photometry from the 2MASS point source catalog. Our new observations, presented in Sect. 2, cover the interval between 0.6 and $0.96 \mu \mathrm{m}$ and, when combined with $\mathrm{JHK}_{S}$ photometry from 2MASS, provide a good sampling of the spectral energy distribution in the region around its peak for young low mass stars and brown dwarfs. The wide wavelength baseline allows us to perform a robust fit to the intrinsic spectral energy distribution predicted by model atmospheres and pre-main sequence evolutionary tracks, which we describe in Sect. 3. In this way we produce lists of candidate members of the star-forming region, which can be compared to the census of members obtained by previous works like those outlined above using different approaches.
A major uncertainty factor in deriving intrinsic properties of young stellar objects is the rather poor accuracy with which the distances to their host star-forming regions are generally known. In the case of Lupus, the existent distance determinations have been reviewed by Comerón (2008). Following the discussion in that work we will adopt 150 pc for Lupus 1 and 4, and 200 pc for Lupus 3.

The areas covered by the survey presented here were chosen so as to have a large overlap with the regions observed by the Spitzer legacy program From molecular cores to planet-forming disks, and data presented here have already been used in the analyses of Merín et al. (2007) and Merín et al. (2008). Therefore, this paper provides the detailed description of the visible observations used in those works.

\section{Observations and data reduction}

The material presented in this paper is based on imaging of the Lupus 1, 3, and 4 clouds obtained with the Wide Field Imager (WFI) at the MPI-ESO 2.2m telescope on La Silla, Chile (Baade et al. 1999). Images were obtained through filters approximately covering the Cousins $R_{\mathrm{C}}$ and $I_{\mathrm{C}}$ bands, as well as through an intermediate-width band near $0.96 \mu \mathrm{m}$ to which we will refer as zwFI. The detector plane of WFI is covered by an array of $4 \times 2$ individual CCD chips, each with $2048 \times 4096$ pixel $^{2}$, covering a field of view of $34^{\prime} \times 33^{\prime}$ with small gaps of $14^{\prime \prime}$ and $23^{\prime \prime}$ between adjacent chips. The pixel scale is $0^{\prime \prime} 238$ pixel $^{-1}$.

A total of 24 fields were observed covering the areas of highest dust column density in each of the clouds according to IRAS $100 \mu \mathrm{m}$ emission maps, selected to include most of the areas surveyed in the Spitzer Legacy program From molecular cores to planet-forming disks. The area of the sky covered in each cloud is 2.84 square degrees in Lupus 1, 2.78 in Lupus 3, and 1.11 in Lupus 4. The observations were carried out in Service Mode during ESO periods 69 (April-October 2002) and 71 (April-October 2003). Since the execution in Service Mode implies that the observations are carried out when the external conditions are within a pre-specified range, rather than on fixed dates (Comerón 2004), individual observations were spread over many observing nights, spanning a few months every period. The observations scheduled in 2003 included fields that could not be observed at all in the previous year, as well as the completion of the observations of fields that could be imaged only in some of the filters in 2002.

The observations of each individual field were distributed in two Observation Blocks (OBs). The first OB described the instrument setup and exposure parameters in the $R$ and $I$ filters, while the second one described the observation in the $z_{\text {WFI }}$ filter. Such split was needed due to the long exposure time per field when adding the integrations in all the filters, and facilitated the scheduling of the observations. The disadvantage is that observations in $R$ and $I$ on one side, and $z_{\text {WFI }}$ on the other, were in general not simultaneous. Furthermore, most of the $z_{\text {WFI }}$ observations were carried out on the second year, whereas the $R$ and $I$ observations were roughly evenly distributed between both periods. As a result, for many fields the lag between the $R I$ and the $z_{\text {WFI }}$ observations can approach or slightly exceed one year. The total exposure times per field and filter were 1600s in $R$, $240 \mathrm{~s}$ in $I$, and $1320 \mathrm{~s}$ in $z_{\mathrm{WFI}}$. The observations in each filter were split into four individual exposures, shifting the telescope pointing by small amounts (between $1^{\prime}$ and $2^{\prime}$ ) between consecutive pointings so as to cover the gaps between the individual chips forming the WFI detector. The total coverage of each field led to an overlap band $5^{\prime}$ wide between adjacent fields. 
The observations were processed by using a number of dedicated IRAF ${ }^{1}$ scripts in charge of the different steps of the data reduction. Master bias frames were constructed, for each night in which observations for our program were obtained, from the standard calibration data products supplied with the Service Mode data package. Regarding flat fields, the best results were obtained with twilight sky flat field frames rather than with dome flat field frames. The stability of the sky flat field frames was found to be sufficiently good for the use of a single master sky flat field frame in each filter for each of the observing seasons, which was obtained by combining all the sky flat field frames included in our data packages.

Fringing strongly affects the WFI observations taken in the $I$ and $z_{\text {WFI }}$ filters. To remove the fringing pattern, we first identified the science frames in those filters in our data sets that were virtually free from nebulosity. After inspection of the fringing pattern we grouped those sharing the same pattern, since $2-3$ pattern changes typically took place during the period spanned by our observations in each of the two seasons. Then, a master fringing frame in each of the two affected filters was constructed by subtracting from each frame its average sky level, and median filtering all the frames of each group together. The suitable master fringing frame was then scaled by a factor of order unity to account for the amplitude of the fringes in the individual science frames. The precise value of the scaling factor was determined by iteratively subtracting from each science frame the master fringing pattern with different values of this factor, until finding one that left no visible residual fringing. We found that visual inspection provided the best criterion to determine the fringing scaling factor. In all cases a good solution could be found that effectively removed the fringing pattern of the science frames after proper scaling.

Another factor affecting WFI frames, common to focal reducers, is the existence of sky concentration, which requires the determination of an illumination correction map. This effect is well known to exist at the WFI to the level of a few hundredths of a magnitude throughout most of the field, reaching slightly above 0.1 mag near the edges of the field. Since no calibrations suitable for a quantification of this effect in our images were obtained, we applied instead the WFI illumination correction map in the $R$ band derived by Koch et al. (2003) and assumed it to be approximately applicable to the $I$ and $z_{\text {WFI }}$ filters as well. Judging from the residual differences between the illumination correction maps computed by Koch et al. in different filters, we expect the systematic error introduced in this way not to exceed $0.03 \mathrm{mag}$ anywhere in the field. On the other hand, we examined the residuals of the fits of photometric solutions derived from observations of Landolt (1992) standard star fields (Sect. 2.1) obtained as a part of the WFI calibration plan during the nights in which our observations were carried out. Although the crowding of these fields with standard stars is far too sparse to independently derive illumination correction maps, the absence of systematic positional trends in the residuals gives us confidence that the applied procedure is indeed sufficiently accurate down a few hundredths of a magnitude.

Once corrected for bias, flat field, fringing and illumination, individual images in each filter were combined into a single mosaic per field. This was done by determining the offsets among the four exposures of each field for each individual detector chip using the stellar images as references, and then combining the

${ }_{1}$ IRAF is distributed by NOAO, which is operated by the Association of Universities for Research in Astronomy, Inc., under contract to the National Science Foundation. images in each chip correcting for the offsets. Because the amplitude of the offsets was larger than the size of the interchip gaps, the combined, offset-corrected images of each chip overlapped, thus making it possible to precisely determine the offsets between adjacent chips using the stars in the overlap regions as reference. In this way a single full field image was produced for each filter by stacking together the four individual exposures.

Astrometric calibration of each field was carried out by using stellar positions in the USNO-A2.0 catalog, which provides several thousand stars per field. A distortion correction was then applied to the images through scripts making use of the IRAF GEOMAP and GEOTRANS tasks. The rms error in stellar coordinates is found to be better than $0^{\prime \prime} 3$ by comparison to the USNO-A2.0 astrometry, and confirmed by cross-matching with the 2MASS point source catalog (see Sect. 2.1.3).

The distortion-corrected images of each field in the three observed filters were stacked together into a single combined image where source detection was carried out using DAOFIND. Aperture photometry, using IRAF scripts based on DAOPHOT (Stetson 1987) on each position where a source was detected, was then carried out on the original combined image in each filter, thus producing a catalog with the coordinates and instrumental magnitude of each object in each of the three filters. The aperture radius was individually set for each image taking as a reference the full-width at half-maximum (FWHM) of bright unsaturated stars, in order to account for the varying image quality among the different fields. An aperture radius of five times the FWHM (i.e. typically about $6^{\prime \prime}$ ) was used.

\subsection{Photometric calibration}

\subsection{1. $R_{\mathrm{C}}$ and $I_{\mathrm{C}}$ filters}

At least one, and frequently more, observations of Landolt standard star fields (Landolt 1992) were carried out every night that $R$ and $I$ observations were obtained for our program. This allowed us to determine both photometric zeropoints and color terms for each of those two filters in order to convert instrumentally determined magnitudes to the Cousins system. No attempt was made to determine in addition extinction terms, since the small number of standard star fields observed per night prevented their determination on a nightly basis. Instead, average extinction coefficients for La Silla in those filters were used.

Whereas the $R$ and the $z_{\text {WFI }}$ filters used were the same in the 2002 and 2003 periods, the standard $I$ filter used at WFI, denominated $I c$ in 2002, was replaced shortly after the beginning of the 2003 period by the so-called $I / 203$ filter. As a result, part of our observations carried out in 2003 were obtained with a $I$ filter different from that used for the rest of the observations. Comparing the zeropoints obtained from the analysis of the Landolt standard star fields with both filters, we find that observations with the $I / 203$ filter are approximately 0.3 mag deeper. Furthermore, the $I / 203$ filter is closer to the Cousins $I_{C}$ filter, as shown by the lower value of the color terms. These were determined by solving the following equations for each standard star field observed:

$$
\begin{aligned}
R_{\mathrm{C}} & =r+k_{r}^{\prime \prime}\left(R_{\mathrm{C}}-I_{\mathrm{C}}\right)+C_{r}+k_{R}^{\prime} X \\
I_{\mathrm{C}} & =i_{203}+k_{i_{203}}^{\prime \prime}\left(R_{\mathrm{C}}-I_{\mathrm{C}}\right)+C_{i_{203}}+k_{I}^{\prime} X \\
I_{\mathrm{C}} & =i_{\mathrm{C}}+k_{i_{C}}^{\prime \prime}\left(R_{\mathrm{C}}-I_{\mathrm{C}}\right)+C_{i_{C}}+k_{I}^{\prime} X
\end{aligned}
$$

where $r, i_{203}$, and $i_{c}$ are instrumental magnitude measurements in the $R, I / 203$, and $I c$ filters; $C_{r}, C_{i_{203}}$, and $C_{i_{\mathrm{C}}}$ denote zeropoints in those filters; $k^{\prime}$ are the extinction terms; $X$ is the airmass; and $k^{\prime \prime}$ are the color terms. From the standard stars included in each 
Table 1. Saturation limits and limiting magnitudes at $3 \sigma$ level.

\begin{tabular}{ccc}
\hline \hline Filter & Mag Sat. & Mag $3 \sigma$ \\
\hline$R c / 162$ & 14.5 & 24.5 \\
$I / 203$ & 11.8 & 22.5 \\
$I c$ & 11.5 & 22.2 \\
$z_{\text {WFI }}$ & 10.5 & 22.0 \\
\hline
\end{tabular}

field, the following average values for the color term coefficients were obtained:

$$
\begin{aligned}
k_{r}^{\prime \prime} & =-0.054 \pm 0.070 \\
k_{i_{203}}^{\prime \prime} & =0.027 \pm 0.051 \\
k_{i_{\mathrm{C}}}^{\prime \prime} & =0.208 \pm 0.097 .
\end{aligned}
$$

These average values were then used to recompute the nightly zeropoints for each individual field.

Since the goal of our observations is to characterize the lowmass population of the Lupus clouds, the long exposure times used lead to detector saturation even at relatively faint magnitudes. Table 1 lists both the saturation magnitudes in each filter and approximate $3 \sigma$ detection limits. Moderate variations within the $0.2 \mathrm{mag}$ level in these values occur from field to field due to the different external conditions under which our observations were carried out.

\subsection{2. zWFI filter}

The calibration of the $z_{\text {WFI }}$ filter was carried out based on observations of the star Wolf 629, which has been adopted as a standard both in the Johnson-Cousins system, and in the Gunn system used by the Sloan Digital Sky Survey (Fukugita et al. 1996). A cycle of observations consisting of short $z_{\text {WFI }}$ exposures of the field around Wolf 629 immediately followed by also short exposures at the centers of the Lupus 1, 3, and 4 areas in the same filter was obtained in order to set up a network of secondary standards in each of the individual clouds. Each of the deep fields imaged in $z_{\text {WFI }}$ was then calibrated with reference to this shallow exposure. For the fields where an overlap region with the shallow exposure on the center of the field existed, bright, unsaturated stars in the area of overlap were used. For fields farther from the cloud center not overlapping with the shallow reference exposure, cross-calibration was achieved by using stars in the overlap region with contiguous fields, which typically provided a few hundred suitable stars in common.

The $z_{\text {WFI }}$ filter is characterized by an effective wavelength of $957 \mathrm{~nm}$, which is somewhat longer than that of the Gunn $z^{\prime}$ filter $(911.4 \mathrm{~nm})$, and a width of $53.4 \mathrm{~nm}$. Using the STIS spectrophotometric calibration of Vega (Bohlin \& Gilliland 2004), the flux at zero magnitude at the effective wavelength of the $z_{\mathrm{WFI}}$ filter was determined to be $f_{957}^{0}=7.44 \times 10^{-13} \mathrm{~W} \mathrm{~cm}^{-2} \mathrm{~s}^{-1} \mu \mathrm{m}$. The magnitude of Wolf 629 in that filter was then determined using its Gunn $r^{\prime}, i^{\prime}$, and $z^{\prime}$ photometry as given in Smith et al. (2002) and a template M4 star spectrum, thus obtaining $z_{\mathrm{WFI}}=8.361$.

\subsubsection{Final catalog including $2 \mathrm{MASS}$ data}

Three merged catalogs (one for each of the observed clouds) with position and photometry, as well as the date of the observation in each filter, were produced. For each star appearing in more than one field, the entry corresponding to the field where its angular distance to the field center was smaller was retained, rather than averaging the magnitudes derived in each field. This
Table 2. Number of sources for which photometry is available.

\begin{tabular}{lccc}
\hline \hline Cloud & $R, I$, or $z_{\mathrm{WFI}}$ & $R I z_{\mathrm{WFI}}$ & $R I z_{\mathrm{WFI}} J H K$ \\
\hline Lupus 1 & 329252 & 220967 & 37523 \\
Lupus 3 & 742411 & 578591 & 82981 \\
Lupus 4 & 328336 & 234706 & 36911 \\
\hline
\end{tabular}

was preferred in order to reduce the effects of the higher uncertainty of the illumination correction towards the edges of the fields.

Each entry in the catalog was further complemented with near-infrared $J H K_{S}$ photometry by cross-matching it with the 2MASS Point Source Catalog (Skrutskie et al. 2006). A matching radius of $2^{\prime \prime}$ was defined, which appears to be by far sufficient given that in all fields over $94 \%$ of the matches corresponded to differences between the 2MASS positions and those determined by us of less than 1". When more than one 2MASS source was found within the $2^{\prime \prime}$ circle, the one closer to the position derived from our observations was chosen as the counterpart.

Table 2 gives bulk numbers reflecting the contents of our catalogs. In total, our observations contain nearly 1.4 million objects for which the magnitude in at least one of the $R, I$, or zWFI filters has been measured, and 157415 for which six-band photometry from 0.6 to $2.2 \mu \mathrm{m}$ is available. The numbers given in Table 2 show the ability of our observations to image sources too faint to be detected by 2 MASS, as only $15 \%$ of the sources that we detect in all three WFI filters have a counterpart detected in 2MASS. The catalogs used in this study are available from the authors upon request.

\section{Analysis}

The multiband photometry available from the merging of our observations and the 2MASS Point Source Catalog enables a highly reliable determination of the spectral energy distributions of a vast number of objects covering a sizeable fraction of the volume of the star-forming regions in Lupus 1,3 and 4 . The available wavelength range from the $R_{\mathrm{C}}$ to the $K_{S}$ bands samples the peak emission of cool, low mass objects. We thus expect the flux in all these bands to be generally dominated by photospheric emission, although exceptions due to veiling, emission lines or warm circumstellar dust may exist as discussed in Sect. 4. The long wavelength baseline makes it possible to disentangle the intrinsic spectral energy distribution from the reddening effects caused by dust associated to the host clouds along the line of sight. Finally, the effects of extinction in those bands are expected to be relatively small taking into account the moderate extinction levels present in the observed regions (Cambrésy 1999).

As noted in Sect. 2.1.3, the depth of our WFI observations is such that nearly $85 \%$ of the sources detected in them are too faint to appear in the 2MASS catalog. On the other hand, wavelength coverage restricted to the $R_{\mathrm{C}} I_{\mathrm{C}} z_{\mathrm{WFI}}$ bands is too limited to carry out a reliable simultaneous fit of the spectral energy distribution and the foreground reddening, thus preventing us from determining the characteristics of these faint sources in the same way as we do for those for which photometric data extend to the near-infrared. We thus preferred to confine this study to the portion of our source catalog for which at least complete $I_{\mathrm{C}} z_{\mathrm{WFI}} J H K_{S}$ coverage is available, as our results indicate that a fit in the $0.8-2.2 \mu \mathrm{m}$ interval is the minimum required for a reliable determination of the intrinsic spectral energy distribution. 

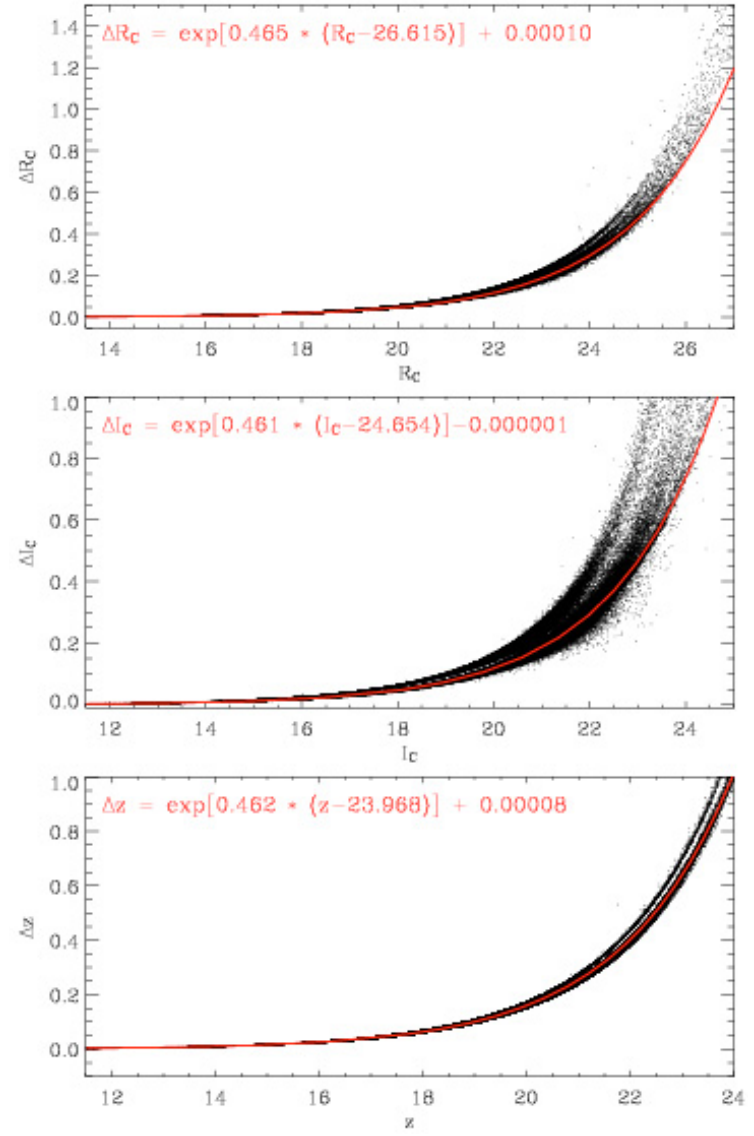

Fig. 1. Photometric errors versus magnitudes and relative exponential fits for all the point-like sources detected in Lupus 3. The "multiple sequence" is due to different seeing conditions during the observation of the different fields.

Whenever available we also use the flux measured at $R_{\mathrm{C}}$ to improve the reliability of the fit. The requirement of having 2MASS measurements available is likely to leave out of the current analysis some interesting very low mass members of the Lupus clouds, which we are not able to reliably identify as such on the basis of the $R_{\mathrm{C}} I_{\mathrm{C}} z_{\mathrm{WFI}}$ data alone. The identification of the lowest-mass population of the clouds potentially detected in our WFI survey using the same methodology described in this paper is thus deferred to further work making use of deeper near-infrared photometry.

Although limited to sources for which photometry between $0.6 / 0.8$ and $2.2 \mu \mathrm{m}$ is available, the sensitivity of the combined WFI and 2MASS data is sufficient to probe deep into the substellar mass function of the Lupus clouds. At the 2MASS limiting magnitude of $K_{S} \simeq 15.5$, assuming as typical parameters for a source in Lupus 3 (probably the most distant of the three clouds) a distance of $200 \mathrm{pc}$, an age of $5 \mathrm{Myr}$, and an extinction $A_{V}=5 \mathrm{mag}$, evolutionary models indicate that our survey should be able to detect brown dwarfs with masses down to $0.02 M_{\odot}$ (Baraffe et al. 2003), having temperatures as low as $2400 \mathrm{~K}$ corresponding to a spectral type L0-L1 (Kirkpatrick 2005), and even cooler and later objects if their age is younger. On the other hand, for lightly reddened members the saturation limit is first reached in the $R$ band, with the limit of $I_{\mathrm{C}}=11.8$ given in Table 1 corresponding to an unreddened star of $0.6 M_{\odot}$ and 5 Myr age at the distance of the nearest cloud, Lupus 1, with a temperature of $\simeq 3600 \mathrm{~K}$ and spectral type M1 (Luhman et al. 2003). While the actual limits vary depending on the actual age, distance, and extinction, the dynamic range of the merged WFI/2MASS photometry thus samples well the very low mass stellar and massive brown dwarf domains and the early-M to early-L spectral type interval.

\subsection{Temperature and luminosity fits}

The procedure that we used to estimate the individual temperature and luminosity of each object is based on the simultaneous fit of all the measured broad-band fluxes to model spectra of cool stellar and substellar photospheres, and is similar to that used by Spezzi et al. (2007) in their study of the stellar population in Chamaeleon II. The grid of reference model spectra used is that of Hauschildt et al. (1999) and Allard et al. (2000), covering the effective temperature $\left(T_{\text {eff }}\right)$ range $1700 \mathrm{~K}-10000 \mathrm{~K}$. A single value of the surface gravity, $\log g=4$, was chosen as being representative of very low mass stars and brown dwarfs, since broad-band colors have only a mild dependency on the actual surface gravity in this range. Using the flux per unit of stellar surface provided by the synthetic spectra, absolute magnitudes in each of the filters used, $M_{\text {synt }}^{\lambda}$, are obtained for fiducial stars of different $T_{\text {eff }}$, each with a radius arbitrarily set to $1 R_{\odot}$. The observed magnitude $m_{\mathrm{obs}}^{\lambda}$ at each band can then be expressed as

$m_{\mathrm{obs}}^{\lambda}=M_{\mathrm{synt}}^{\lambda}+A_{V} \cdot \frac{A_{\lambda}}{A_{V}}+S$

where $A_{V}$ is the foreground extinction in the visible, $A_{\lambda}$ is the corresponding extinction at wavelength $\lambda$, and $S$ is a wavelength-independent scaling factor that contains the dependency on the actual distance $D$ and radius $R$ of the star:

$S=5 \log D(\mathrm{pc})-5 \log R\left(R_{\odot}\right)-5$.

The ratio $A_{\lambda} / A_{V}$ appearing in Eq. (4) can be treated as a constant given by the extinction law, for which we adopt that derived by Cardelli et al. (1989) with a total-to-selective extinction ratio $R_{V}=3.1$. Since the vast majority of sources in the area of our survey are located outside high extinction areas, the choice of this value of $R_{V}$, which is appropriate for the diffuse interstellar medium, appears to be appropriate. However, $R_{V}$ increases inside dense clouds due to a larger grain size cutoff (e.g. Vrba \& Rydgren 1984; Steenman \& Thé 1989), which makes the wavelength dependency of the extinction shallower. We estimate the systematic effects introduced by our adoption of $R_{\mathrm{V}}=3.1$ by comparing the extinction curves of Weingartner \& Draine (2001) for $R_{\mathrm{V}}=3.1$ and $R_{\mathrm{V}}=5.5$, the latter value being representative of embedded star-forming regions, and we derive the amount of extinction needed in either case to produce a given amount of reddening on the spectral energy distribution of an embedded object. In the wavelength range covered by our observations, the extinction $A_{\mathrm{V}}$ needed to produce a given amount of reddening with $R_{V}=5.5$ is increased by $10 \%$ with respect to the extinction yielding the same color excess. As will be shown in Sects. 4 and 5 , we obtain $A_{\mathrm{V}} \simeq 10$ mag for the most obscured objects to which we apply our method. If $R_{\mathrm{V}}=5.5$ were the correct characterization of the extinction curve for those, our luminosity estimate with $R_{\mathrm{V}}=3.1$ would lead to an underestimate of the luminosity by a factor of 2.5 . While significant, such underestimate is rather modest when considering the dramatic variations in luminosity that take place along pre-main sequence evolution. For instance, at $T_{\text {eff }}=3000 \mathrm{~K}$, the luminosity ratio between objects of ages $1 \mathrm{Myr}$ and $5 \mathrm{Myr}$ is more than twice that value. Furthermore, both the assumptions of $R_{\mathrm{V}}=5.5$ and of $A_{\mathrm{V}}=10$ used to illustrate this point are somewhat extreme, since the need 
to detect the sources at visible wavelengths in order to fit their spectral energy distribution limits our study to generally more lightly embedded objects (smaller $A_{\mathrm{V}}$ ) and to less dense regions of the molecular clouds (smaller $R_{\mathrm{V}}$ ).

Assuming a certain input value of $T_{\text {eff }}$ for a star, one equation like Eq. (4) can be set for each photometric band. These equations form an overdetermined system with the two unknowns $A_{\mathrm{V}}$ and $S$, which can be solved by least squares. The temperature that minimizes the residual of the fit is then taken as the temperature of the object, and the luminosity $L$ is derived from the value of $S$ obtained for the best fitting temperature as $L=4 \pi \sigma R^{2} T_{\text {eff }}^{4}$, where $\sigma$ is the Stefan-Boltzmann constant. The residuals for this best fit give in turn an estimate of the quality of the solution. It should be in principle possible to obtain a more reliable fit by weighting each equation by the inverse of the magnitude uncertainty, $\sigma_{\lambda}^{-1}$. However, we preferred not to use such weights, which are composed of the random measurement error and a possible systematic error caused by shortcomings in the models. The latter is poorly known, but it is likely to dominate especially at infrared wavelengths where the objects are brightest and the magnitude uncertainty is very small. We carried out numerical experiments comparing the results obtained for known cloud members with well constrained temperatures, where we computed their temperatures by solving the system of Eqs. (4) either without weighting them, or by weighting them with the inverse of the photometric uncertainty alone. In general we find a better match in the non-weighted results, seemingly confirming our suspicion that systematic errors dominate over the photometric ones.

The best-fitting $T_{\text {eff }}$ derived for each star, and the corresponding values of $A_{\mathrm{V}}$ and $S$, are expected to be close to their true values regardless of whether or not the object is a member of the star-forming region, since the colors of young stellar objects, main sequence late dwarfs, and cool giants of a given temperature are to a first approximation similar. On the other hand, it is straightforward to transform $S$ into the luminosity if the object is a member of the star-forming region. For background and foreground members, the luminosity remains however undetermined due to their unknown distances.

The suitability of the wavelength range used to sample the spectral energy distribution of the objects of interest is illustrated in Fig. 2, where we show the fit of the photometric data points to the reddened synthetic spectrum of a $T_{\text {eff }}=3000 \mathrm{~K}$ photosphere. The pronounced change of slope of the spectral energy distribution in the region around its peak allows us to reliably disentangle the effect of temperature from that of extinction.

The residuals of the spectral energy distribution fits are used to estimate their quality. After producing fits for all the sources in our sample, we find that $87 \%$ of them yield averages residuals below $0.2 \mathrm{mag}$, which we consider of good quality in view of the accuracy of our photometry. The percentage actually varies between $86 \%$ for Lupus 3 and 4, and $91 \%$ for Lupus 1 . The reason is mainly the higher galactic latitude of the latter, which leads to a smaller number of close pairs not disentangled by our automatic photometry. Poor fits are also obtained for variable sources due to the non-simultaneity of the photometry, as we discuss in Sect. 4. The extinction is derived to a typical accuracy of $\Delta A_{V}=0.15 \mathrm{mag}$, and the luminosity to $\Delta \log L=0.03$. As discussed in Sect. 4 , the accuracy in the determination of the temperature is estimated to be $\pm 200 \mathrm{~K}$ in the range of temperatures of interest, $T_{\text {eff }}<4000 \mathrm{~K}$.

It must be stressed that, whereas the method described here can be efficiently used to derive parameters of cool stars in the field, it is not intended to provide by itself a membership

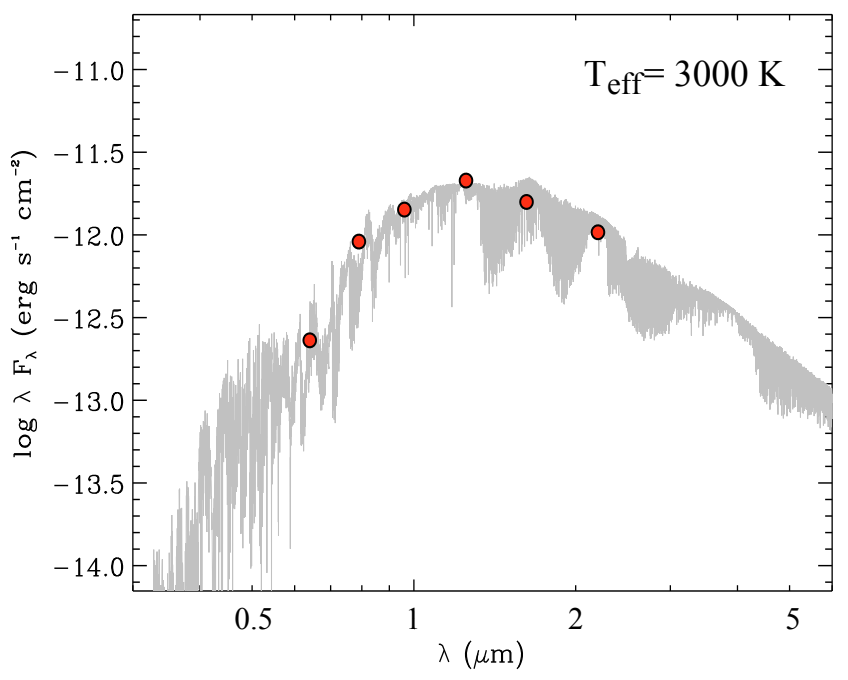

Fig. 2. Example of the fit of the $R_{\mathrm{C}} I_{\mathrm{C}} z_{\mathrm{WFI}} J H K_{S}$ photometry (dots) to a synthetic spectrum (grey line). In this case, the model providing the best fit corresponds to a temperature of $3000 \mathrm{~K}$. The sampling around the peak of the spectral energy distribution allows us to determine the temperature to an accuracy of few hundreds K, as discussed in Sect. 4.

criterion. However, under certain circumstances that we describe in Sect. 5 the value of $S$ can be used as a strong indicator of membership within a certain range of distances and ages.

\subsection{Estimating the contamination}

The derivation of the best-fitting values of $T_{\text {eff }}$ and $S$ for each star allows us to plot them in a diagram that is closely linked to the commonly used $L$ vs. $T_{\text {eff }}$ diagram in the case of members of the star-forming region. It is also easy to convert theoretical isochrones and evolutionary tracks so that ages and masses of the members can be determined by relation to them. In this diagram, the isochrones delimiting the age range set by the time span in which the aggregate has been forming stars can be expressed as $S_{1}=S\left(T_{\mathrm{eff}}, t_{\min }\right)$ and $S_{2}=S\left(T_{\mathrm{eff}}, t_{\max }\right)$, where $t_{\min }$ and $t_{\max }$ are respectively the ages of the youngest and oldest members of the aggregate. All members of the aggregate are thus expected to be found in the band defined by these isochrones. This is illustrated in Fig. 3, where the position of all the stars for which $T_{\text {eff }}$ and $S$ can be determined in the field of Lupus 3 are plotted together with two representative isochrones. For the latter we used the evolutionary tracks of Baraffe et al. (1998) for stellar masses down to $0.075 M_{\odot}$, and the models allowing dust formation in the photosphere of Chabrier et al. (2000) for substellar masses.

The band occupied by members of the aggregate may be expected to be populated also by foreground and background stars of similar temperature, whose $D / R$ ratios lead to a value of $S$ within the same range. In the cool temperature range of interest in this work, these potential contaminants are split into two well separated categories with similar temperatures but very different luminosities. Foreground sources are expected to be main sequence cool dwarfs, whereas red giants are expected to dominate the background population. This is most clearly seen at the lowest temperatures shown in Fig. 3.

\subsubsection{Main sequence contamination}

To estimate the number of main sequence contaminants we used the log-normal local initial mass function of Chabrier (2005), 


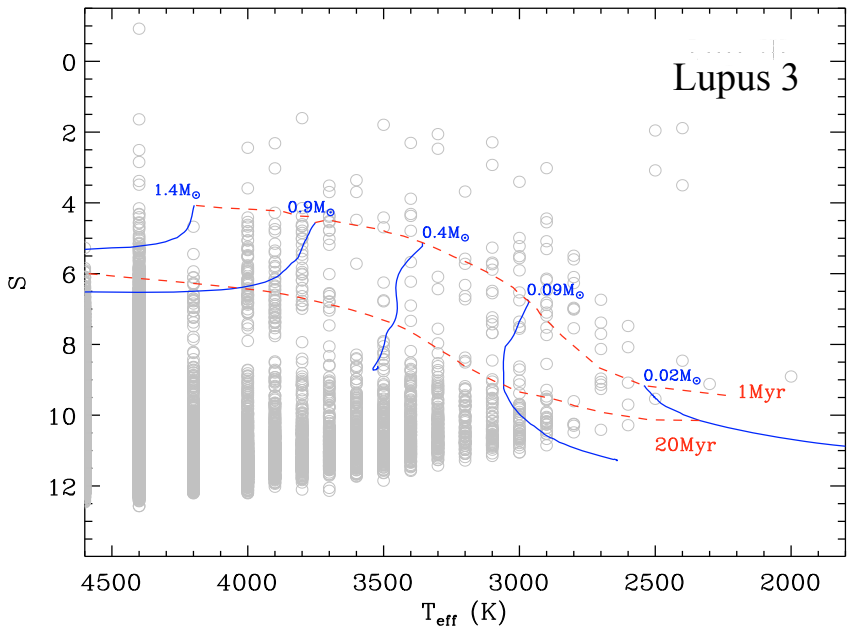

Fig. 3. $S$ vs. $T_{\text {eff }}$ for the Lupus 3 region, showing the positions of all the stars in our images for which a temperature below $4500 \mathrm{~K}$ is derived. Two isochrones defining the locus occupied by members of Lupus 3 with ages between 1 and $20 \mathrm{Myr}$ are plotted, as well as evolutionary tracks for stars and brown dwarfs of different masses at the distance of Lupus. The figure illustrates that the vast majority of cool objects detected in our images are field dwarfs characterized by higher values of $S$, thus falling below the band where Lupus 3 members should be found.

assuming that all foreground stars at a given $T_{\text {eff }}$ are on the main sequence and thus have a well-defined $T_{\text {eff }}$ vs. mass relationship, which we derive from the $5 \mathrm{Gyr}$ isochrone from Baraffe et al. (1998). The number of stars per logarithmic mass $(M)$ interval that enter the region between $S_{1}$ and $S_{2}$ in an area of the sky that subtends an angle $\Omega$ is then

$\frac{\mathrm{d} N(M)}{\mathrm{d} \log M}=\frac{1}{3} \xi(\log M) R^{3}(M) 10^{3}\left[10^{0.6 S_{2}}-10^{0.6 S_{1}}\right] \Omega$

where $R(M)$ is the stellar radius in units of $R_{\odot}$, and $\xi(\log M)$ is the log-normal branch of the local initial mass function of Chabrier (2005), valid for masses below $1 M_{\odot}$ :

$\xi(\log M)=0.093\left(\log M_{\odot}\right)^{-1} \mathrm{pc}^{-3} \exp \left(-\frac{(\log M-\log 0.2)^{2}}{2 \times(0.55)^{2}}\right)$

with the mass given in $M_{\odot}$ units. To a first approximation, the main sequence defined by field stars placed at a certain distance from the Sun runs roughly parallel to the young isochrones delimiting the locus occupied by Lupus members. Therefore, the foreground stars that appear in that locus are all within a thick shell around the Sun, whose inner and outer radii depend on the distance to the clouds and the limiting isochrones, and only weakly on the spectral type. Stars at shorter distances than the inner edge of the shell appear brighter than the youngest cloud members of the same temperature.

Some illustrative results are presented in Table 3, where the number of contaminants expected up to a certain temperature in each region is given. The upper part of the table shows that the number of foreground stars with both $T_{\text {eff }}$ and $S$ in the range covered by members of the clouds with ages between 1 and $20 \mathrm{Myr}$ is in general small. The expected foreground contamination in Lupus 3 is somewhat stronger due to the greater distance assumed for this cloud, which moves the isochrones down by $0.6 \mathrm{mag}$ in $S$. However, this is also the most densely populated cloud according to previous studies, and the contamination is thus lower in relative terms.

\subsubsection{Background cool stars}

We estimated the expected level of background contamination using the volume density laws for the different galactic structural components described by Wainscoat et al. (1992). Red giant stars dominate the background contribution to the star counts in the direction of Lupus. We adopted for these stars the effective temperatures and radii from Fluks (1998). As in the case of foreground stars, background cool giants at a given $T_{\text {eff }}$ and radius appear in the region of the $\left(T_{\text {eff }}, S\right)$ diagram occupied by cloud members if they lie within a range of distances set by those intrinsic properties, the distance of the clouds, and the limiting isochrones of cloud members. To estimate the background contribution we thus integrated the volume density seen in the direction of each cloud over this distance range.

The results are also presented in Table 3 . The most obvious feature when comparing the background contamination towards each cloud is the much higher level expected in Lupus 3 and 4, which is a consequence of their lower galactic latitude ( $b=9^{\circ} 5$ and $b=8^{\circ}$ for Lupus 3 and 4 respectively, as compared to $b=16^{\circ} 5$ for Lupus 1 ). Also noticeable is the great decrease in the number of background stars above the isochrone corresponding to the oldest stars in the clouds when the temperature range is reduced from $T<3500 \mathrm{~K}$ to $T_{\text {eff }}<3000 \mathrm{~K}$. This is due to the fact that red giants in the $3000<T_{\text {eff }}(\mathrm{K})<3500$ range (corresponding to early $\mathrm{M}$ spectral types) having values of $S$ in the range covered by Lupus cloud members are located in dense regions of the galactic bulge in that direction. Conversely, as we move towards cooler and intrinsically brighter stars above the oldest limiting isochrone, the contributors with $S$ values in the proper range are located in more remote regions of the Galaxy, where they are rarer. Remarkably, for $T_{\text {eff }}<3500 \mathrm{~K}$ almost no background stars are expected to be found in the locus occupied by Lupus members with ages between 1 and $20 \mathrm{Myr}$, since nearly all such background stars appear much brighter than Lupus members of the same $T_{\text {eff }}$. This is seen in the results of Oliveira et al. (2009), who spectroscopically confirm that the brightest cool stars in the direction of the Serpens star-forming region are background giants. Our case is more favorable in terms of the numbers of such background giants expected, given the higher galactic latitude of Lupus.

We thus conclude that only foreground cool main sequence stars can contribute noticeably to the contamination of the locus of Lupus cloud members, and they are expected to do so in numbers similar to those given in Table 3. A more detailed analysis is presented in Sect. 5, allowing us to produce lists of reliable candidate members of each region.

It must be noted that a particular complication in the interpretation of the census of Lupus is due to the existence of an additional component to those characterized above. By analyzing the results of the ROSAT All-Sky Survey in the direction of Lupus and other regions of the sky and carrying out followup spectroscopy, Krautter et al. (1997) noted the existence of an older, extended population of weak-line $T$ Tauri stars. This population is not particularly concentrated towards the clouds, and an analysis of its large-scale distribution rather links it to the Gould Belt (Wichmann et al. 1997). If the weak-line T Tauri stars in Lupus are at the same distance as the Lupus clouds, as suggested by Wichmann et al. (1997), their older ages should place them below the oldest limiting isochrone, thus making it 
Table 3. Expected numbers of non-members in the Lupus $T_{\text {eff }}-S$ locus.

\begin{tabular}{lcccc}
\hline \hline Cloud & $\begin{array}{c}T_{\text {eff }}<3500 \mathrm{~K} \\
(1<t(\mathrm{Myr})<20)\end{array}$ & $\begin{array}{c}T_{\text {eff }}<3500 \mathrm{~K} \\
\text { (above 1 Myr isochrone) })\end{array}$ & $\begin{array}{c}T_{\text {eff }}<3000 \mathrm{~K} \\
(1<t(\mathrm{Myr})<20)\end{array}$ & $\begin{array}{c}T_{\text {eff }}<3000 \mathrm{~K} \\
\text { (above 1 Myr isochrone) }\end{array}$ \\
\hline Lupus 1 & 9 & 0 & Foreground stars & 0 \\
Lupus 3 & 20 & 1 & 4 & 1 \\
Lupus 4 & 3 & 0 & 9 & 0 \\
Lupus 1 & 0 & 11 & 2 & 0 \\
Lupus 3 & 0 & 110 & Background stars & 3 \\
Lupus 4 & 1 & 40 & 0 & 2 \\
\hline
\end{tabular}

easy to reject them as possible members. However, studies of the distribution of weak-line $\mathrm{T}$ Tauri stars across the Gould Belt by Guillout et al. (1998) rather indicate that these stars span a wide range in distance towards any given direction. Their results further suggest that the Lupus clouds lie near the far end of this distance range in that direction of the sky, with most of the weak-line $\mathrm{T}$ Tauri stars being foreground and extending to distances as close as $30 \mathrm{pc}$ from the Sun. Foreground old T Tauri stars unrelated to the Lupus clouds can thus have $S$ values in the range expected for true cloud members, and thus be photometrically indistinguishable from them. The possible influence of the presence of this component on our results will be discussed in Sect. 6.

\section{The known members of the Lupus clouds}

The many studies carried out over the last decades dealing with the identification and characterization of the stellar and substellar population of the Lupus clouds has provided an abundant census of members. For many of them detailed information on circumstellar environment, intrinsic properties of the central source, or variability is available, thus providing a sound basis to test the validity of the methods outlined in Sect. 3.1.

A compilation of all known members of Lupus 1, 3, and 4, selected by different techniques, has been presented by Merín et al. (2008), who also estimated the spectral type, luminosity, and foreground extinction for most objects based on photometric fits to synthetic spectra, using a procedure similar to ours. The $R_{\mathrm{C}} I_{\mathrm{C}} z_{\mathrm{WFI}} J H K_{S}$ photometry data that they used are the same as in this paper, complemented with data from the literature for saturated sources.

The derived parameters for the known members of each cloud that are detected and unsaturated in at least $I_{\mathrm{C}}$ and $z_{\mathrm{WFI}}$ are presented in Table 4 . It may be noted that Table 4 does not represent a validation of membership based on our new data, but only a derivation of parameters of objects already known to be members from their youth signatures, based on the method described in Sect. 3. For most of the objects we obtain low temperatures, and luminosities and ages consistent with their membership in the Lupus clouds. The vast majority of objects in Merín et al. (2008) are detected in our WFI observations, with some exceptions that we list in Table 5. As expected, the comparison shows that our visible/red survey is particularly incomplete near the high extinction regions of the clouds, most notably Lupus 3, where members are too deeply embedded to be detectable at short wavelengths. Other objects are close to bright sources or their associated nebulosity, outside the field surveyed with WFI or, in a few cases, misclassified as a stellar object whereas the WFI images show it to be a resolved galaxy.
Table 5. Objects classified as candidate Lupus members by Merín et al. (2008) for which no fits are obtained.

\begin{tabular}{ll}
\hline \hline Object (SSTc2dJ) & Notes \\
\hline SSTc2dJ153848.2-344041 & Lupus 1 \\
SSTc2dJ154302.3-344406 & out of the WFI survey field \\
SSTc2dJ154506.3-341738 & in the nebulosity of HT Lup \\
SSTc2dJ154512.9-341731 & very close to HT Lup \\
& Lupus 3
\end{tabular}

SSTc2dJ160708.6-394723 out of the WFI survey field SSTc2dJ160715.2-400342 out of the WFI survey field SSTc2dJ160801.7-391231 galaxy

SSTc2dJ160830.7-382827 out of the WFI survey field SSTc2dJ160834.3-390618 affected by saturation near HR5999/6000 SSTc2dJ160834.6-390534 affected by saturation near HR5999/6000 SSTc2dJ160854.7-393744 out of the WFI survey field SSTc2dJ160858.3-390736 on dark nebulosity, undetected SSTc2dJ160858.3-390749 on dark nebulosity, undetected SSTc2dJ160916.4-390444 on dark nebulosity, undetected SSTc2dJ160918.1-390453 on dark nebulosity, undetected SSTc2dJ160920.3-390402 on dark nebulosity, undetected SSTc2dJ160923.2-390407 on dark nebulosity, undetected SSTc2dJ160926.6-390358 on dark nebulosity, undetected SSTc2dJ160937.2-390407 on dark nebulosity, undetected SSTc2dJ160937.4-390526 on dark nebulosity, undetected SSTc2dJ160939.3-390432 on dark nebulosity, undetected SSTc2dJ161118.7-385824 close to bright star IRAS 16079-3850 SSTc2dJ161222.7-371328 out of the WFI survey field SSTc2dJ161251.7-384216 out of the WFI survey field SSTc2dJ161341.0-383724 out of the WFI survey field Lupus 4

SSTc2dJ155925.2-423507 out of the WFI survey field SSTc2dJ160034.4-422540 out of the WFI survey field SSTc2dJ160044.5-415531 close to bright star MY Lup SSTc2dJ160115.6-415235 on dark nebulosity, undetected SSTc2dJ160221.6-414054 galaxy

The availability of spectral classifications for many of the objects listed in Table 4 provides a check on our derived temperatures. The result of the comparison is presented in Fig. 4, where we also plot the spectral type vs. effective temperature relationships derived by Luhman et al. (2003) for M-type young stellar objects, and by Kenyon \& Hartmann (1995) for main sequence stars of types $\mathrm{K}$ and $\mathrm{M}$. The relative abundance of spectral types later than M0 in the diagram is due to earlier types being generally saturated in our observations, and is not related to the overabundance of mid-M types reported by Hughes et al. (1994). We excluded from the comparison the objects in Table 4 showing evidence of variability as indicated in the notes at the end of the table. Since our observations in $R_{\mathrm{C}}$ and $I_{\mathrm{C}}$ were obtained at epochs generally different from those in $z_{\mathrm{WFI}}$, and were combined with 2MASS $J H K_{S}$ photometry obtained on a yet different epoch, the 


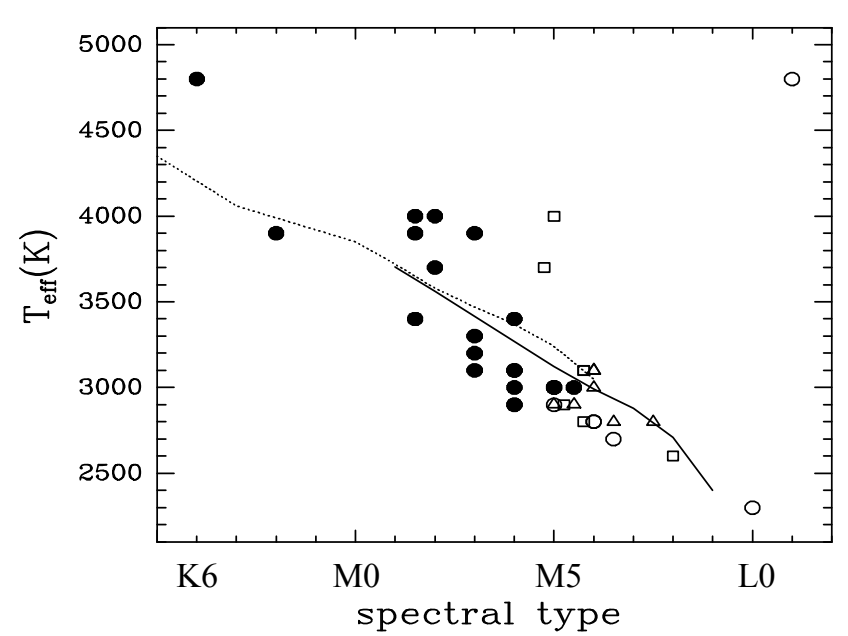

Fig. 4. Comparison between the temperatures obtained with our fitting procedure and the published spectral types of known members of the Lupus clouds (Table 4). Stars known to display photometric variability are excluded from the plot. The outlier near the upper right corner is Lup 654. For SSTc2d160815.0-385715, which is characterized by a noticeable near-infrared excess, we used $T_{\text {eff }}=3700 \mathrm{~K}$ that is obtained by excluding the $K_{S}$ measurement (see Table 7). For Lup 706, the L0 object near the lower right, we used the temperature obtained by excluding the $R_{\mathrm{C}}$ measurement, as justified in the text. The symbols represent the different sources for the spectral type: filled circles from Hughes et al. (1994), open triangles from Comerón et al. (2003), open circles from López Martí et al. (2005), and open squares from other sources given in Table 4. The solid line is the temperature vs. spectral type calibration of Luhman et al. (2003) for pre-main sequence stars and brown dwarfs, and the dotted line is the corresponding calibration by Kenyon \& Hartmann (1995) for main sequence stars.

fit to the spectral energy distribution for variable sources is not meaningful. This is the most likely cause of the relative large fraction of poor fits with a high residual that we obtain among the known members of the clouds.

The appearance of Fig. 4 shows a distinct distribution with most of the objects used for the comparison clustering along the expected spectral type-temperature relationship, whereas some objects appear as clear outliers for which we estimate temperatures well above those expected from their spectral types. Part of the temperature scatter at any given spectral type is undoubtedly due to the limited accuracy with which the temperature can be determined by our fits. However, the different techniques and wavelength ranges used to determine spectral types in different studies are likely to play a role as well. We note in this regard that most of the later stars for which the spectral type is taken from Hughes et al. (1994) lie below the spectral type vs. $T_{\text {eff }}$ calibration. As shown by Comerón et al. (2003), the spectral types in that study tend to systematically differ by about 2 subtypes from those determined by Hughes et al. (1994), in the sense of the Comerón et al. (2003) types being later. The latter authors tentatively attribute the difference to their use of a redder spectral region, less affected by veiling and more suitable for classification based on the shapes of the TiO and VO molecular bands. The application of a correction by two spectral subtypes to the classifications of Hughes et al. (1994) indeed improves their agreement with the calibration beyond M3. On the other hand, we include in Table 4 the classification of López Martí et al. (2005) that is based on intermediate-band photometry of a single selected temperature-dependent spectral feature, being therefore less accurate than an actual spectral classification.
The comparison to the calibrations suggests that the spectral types assigned by López Martí et al. (2005) tend to be only slightly earlier than corresponding to the actual temperature. Spectra obtained from the other sources listed at the bottom of Table 4 are in general in better agreement with the calibration.

The outliers in Fig. 4 are Lup 654, classified by López Martí et al. (2005) as L1 but for which we fit a temperature of $T_{\text {eff }}=$ $4800 \mathrm{~K}$; and, to a lesser extent, the M 5 star SSTc2dJ160853.7391437 , for which we obtain $T_{\text {eff }}=4000 \mathrm{~K}$. No variability information exists for either of these objects, which leaves variability as a possible reason for a temperature overestimate as discussed later in this Section. The rather high values of extinction needed to fit the spectral energy distribution of both object, particularly SSTc2dJ160853.7-391437, hint at an overestimate of the temperature by our method. For this latter object, Allen et al. (2007) note the presence of emission lines, which may hint at veiling or the contribution of the $\mathrm{H} \alpha$ emission to the $R_{\mathrm{C}}$-band flux as another possible cause for an incorrect fit. Finally, a rather large departure from the spectral type vs. temperature calibration is noted for SSTc2d160815.0-385715, an object for which Allen et al. (2007) note a strong near-infrared excess which is supported by our fits when the $K_{S}$ measurement is excluded. The still significant deviation from the expected temperature for its M4.75 spectral type may indicate that other effects may be still present. Still, a low-residual fit $(<0.2 \mathrm{mag} \mathrm{rms})$ can be attained for these three objects.

Among the objects classified as members in Table 4, we find some whose fitted luminosities appear to be inconsistent with membership in Lupus, apparently placing them near or below the main sequence. Most are located in Lupus 3, most likely just because of a size-of-the-sample effect since that cloud contains by far the richest aggregate. Out of the 86 Lupus 3 members for which we obtain a fit, nearly one third (26) are in this category. In at least two cases, Sz 102 and Par-Lup3-4, their underluminosity is well known. It may be explained by the fact that these objects are surrounded by abundant circumstellar material and display jets moving in a direction that is close to the plane of the sky, suggesting substantial obscuration of the central object by a disk seen close to an edge-on orientation (Graham \& Heyer 1988; Fernández \& Comerón 2005).

Another possible explanation is that the spectral energy distributions of some other objects may be affected by the signatures of accretion and warm circumstellar dust in the visible (veiling and strong emission lines) and near-infrared (infrared excess), leading to an erroneous estimate of the temperature. In both situations, our fits tend to overestimate both the temperature and the extinction. We thus produced fits to all the objects in Table 4 excluding the data points in the $R_{\mathrm{C}}$ and $K_{S}$ bands, respectively. Significantly cooler temperatures are estimated for 7 objects when the $R_{\mathrm{C}}$ band is excluded from the fit, as listed in Table 6. The peculiar objects Sz 102 and Par-Lup-4 are among them. Furthermore, the new parameters derived for four of the objects place them above the main sequence. The most remarkable one is Lup 706 (SSTc2dJ160837.3-392311), which is best fitted as a very young brown dwarf (age $<1 \mathrm{Myr}$ ) with $T_{\text {eff }}=2300 \mathrm{~K}$ when the $R_{\mathrm{C}}$ measurement is excluded, in excellent agreement with the independent spectral type estimate of López Martí et al. (2005). Similarly, a good fit is now obtained for SSTc2dJ160927.0-383628, whose derived age $(2.8 \mathrm{Myr})$ places it now in the same range as most of the Lupus 3 population. Two other objects, SSTc2dJ160754.7391545 and SSTc2dJ160708.6-391407, now appear above the main sequence, but still with ages much older than those expected for Lupus 3 members. The low luminosities still obtained 
Table 6. Fits to $I_{\mathrm{C}} z_{\mathrm{WFI}} J H K_{S}$ photometry of stars with suspected veiling.

\begin{tabular}{ccccccc}
\hline \hline Object (SSTc2dJ) & $T_{\text {eff }}$ & $L\left(L_{\odot}\right)$ & $A_{V}$ & $M\left(M_{\odot}\right)$ & Age (Myr) & Notes \\
\hline $160708.6-391407$ & 4000 & 0.086 & 8.1 & 0.62 & 80 & \\
$160754.7-391545$ & 3200 & 0.0037 & 5.0 & 0.13 & 101 & \\
$160829.7-390311$ & 4000 & 0.020 & 1.7 & - & - & 2,4 \\
$160837.3-392311$ & 2300 & 0.0061 & 1.5 & - & $<1$ & 1,2 \\
$160851.4-390530$ & 4000 & 0.020 & 5.9 & - & - & 2,3 \\
$160927.0-383628$ & 3300 & 0.11 & 2.5 & 0.25 & 2.8 & \\
$161204.5-380959$ & 3400 & 0.0033 & 2.6 & - & - & 2 \\
\hline
\end{tabular}

Notes - 1: the fit excluding $R_{\mathrm{C}}$ leads to a good agreement with the very late spectral type (L0) assigned by López Martí et al. (2005); 2 apparently below the main sequence; 3 par-Lup3-4. Best-fitting temperature is still higher than corresponding to its M 5 type (Comerón et al. 2003), possibly due to a combination of veiling, emission lines, the known infrared excess, and variability of this object; 4 Sz 102. The temperature is consistent with the poorly constrained spectral type (K:) proposed by Hughes et al. (1994). The object is well known to be underluminous, perhaps due to blocking due to a disk close to edge-on.

Table 7. Fits to $R_{\mathrm{C}} I_{\mathrm{C}} z_{\mathrm{WFI}} J H$ photometry of stars with suspected infrared excess.

\begin{tabular}{ccccccc}
\hline \hline Object (SSTc2dJ) & $T_{\text {eff }}$ & $L\left(L_{\odot}\right)$ & $A_{V}$ & $M\left(M_{\odot}\right)$ & Age (Myr) & Notes \\
\hline $160703.9-391112$ & 3500 & 0.014 & 2.1 & 0.3 & 101 & \\
$160815.0-385715$ & 3700 & 0.010 & 2.7 & - & - & 1 \\
$161013.1-384617$ & 3200 & 0.0031 & 0.4 & 0.15 & 205 & \\
$161218.5-393418$ & 3800 & 0.014 & 2.5 & - & - & 1 \\
\hline
\end{tabular}

Notes - 1: apparently below the main sequence.

by the best fit to the photometry of these objects, and to the remaining four objects in Table 6 for which no age could be estimated, perhaps indicates that some degree of veiling still subsists at $I_{\mathrm{C}}$, or that other causes such as variability between the different epochs in which the photometry was obtained produce a fit with an incorrect temperature. This is supported by the fact that only a poor quality fit can be obtained for $28 \%$ of the members that apparently lie below the main sequence.

Similar results are obtained when the $K_{S}$ measurement is excluded from our fit to minimize the effect of circumstellar emission in that band. However, the numbers of objects for which the best-fitting temperature substantially changes when excluding the $K_{S}$ measurement is much smaller than those affected by the exclusion of $R_{\mathrm{C}}$. The effect of the $K_{S}$ band exclusion on the derived temperature is typically a change by less than $200 \mathrm{~K}$, even among those objects known or suspected to possess important infrared excesses. For the known members, among which such excesses are expected to be most frequent, best-fitting temperatures obtained in this way are significantly different from those obtained by including the $K_{S}$ measurement in only four cases (including SSTc2d160815.0-385715 as noted above), all of which yield a cooler temperature when the $K_{S}$ measurement is discarded (Table 7). The new positions in the temperatureluminosity diagram rises two of them above the main sequence, but still well below the age strip occupied by most members of Lupus 3.

As a summary of our comparison, both with members of known spectral types and with suspected members still lacking spectral classification, we conclude that our method produces good fits to the spectral energy distribution and reliable estimates of the temperature, luminosity, and foreground extinction towards most of the objects under consideration. A number of discrepancies are found however, either through an estimated temperature significantly different from that expected from the observed spectral type, or through a derived luminosity placing the object near or below the main sequence at the distance of its star-forming region. In a number of cases, the source of the discrepancy can be traced back to variability, suspected veiling or strong emission lines at short wavelengths, or known peculiarities such as an apparent strong underluminosity of the object. It must be noted that, whereas veiling, strong emission lines, and infrared excess as causes of discrepant fits can be suspected by discarding measurements at the shortest and longest wavelengths respectively, variability can be confirmed for only a few objects due to the general unavailability of multi-epoch measurements at either visible or infrared wavelengths. Variability thus remains as just a tentative explanation for the inconsistent fits to the spectral energy distributions of most of the objects.

\section{Evidence for new members}

The distributions in the $S$ vs. $T_{\text {eff }}$ diagram predicted for field low-mass main sequence stars and background cool giants, based on the estimates presented in Sect. 3.2, open up a very useful window for the detection of cool populations associated to the Lupus clouds and, in general, for all the low-mass starforming regions located in the $\sim 100-300 \mathrm{pc}$ distance range from the Sun. This window can be qualitatively understood in the following way: at a given temperature, the number $n(S)$ of field main sequence stars per unit area decreases with decreasing $S$ as $n(S) \propto S^{0.6}$, as can be easily derived from the assumption of a constant volume density of stars and from the definition of $S$. At the distance of Lupus, this results in an almost negligible value of $n(S)$ for temperatures below $\sim 3500 \mathrm{~K}$ for the typical values of $S$ expected among members of the clouds, as shown in Table 3. On the other hand, cool giants in that same range of temperatures have low values of $S$ due to their large radii, unless they are extremely distant and thus located in regions of the Galaxy where the stellar density is exceedingly small. This yields a surface density of cool giants that is significant only for values of $S$ generally lower than those accessible to the pre-main sequence members of the star-forming regions. As a result for $T_{\text {eff }}$ below $\sim 3500 \mathrm{~K}$, the members of the Lupus clouds, and of other starforming regions located at a comparable distance, are largely 

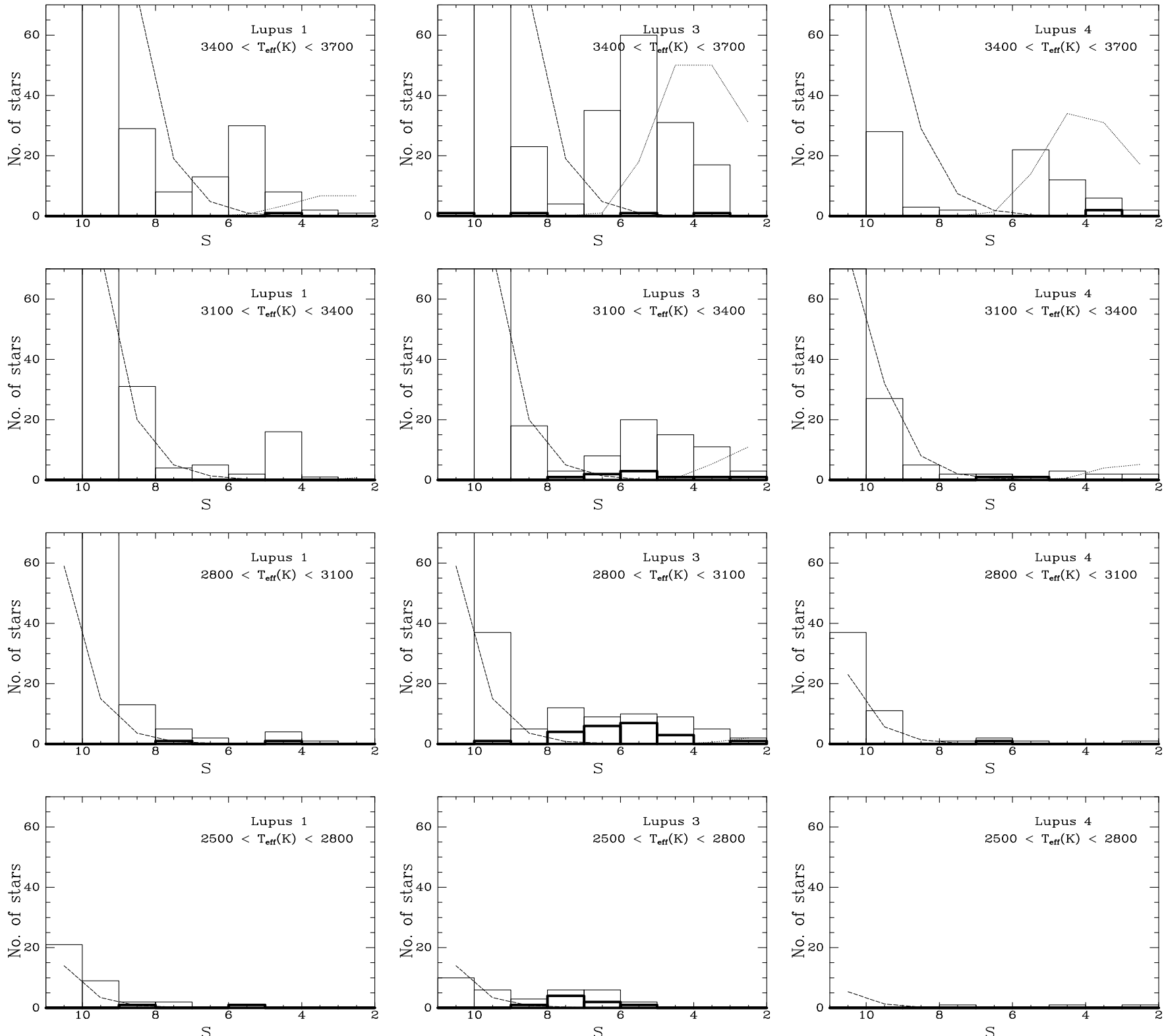

Fig. 5. Histograms of $S$ values obtained at different temperature ranges in each of the observed regions. The thick histogram bars denote the contribution by known members of the star-forming region. The dashed lines indicate the expected contamination by field main sequence stars, and the dotted lines the contamination by background giants. Incompleteness due to the limiting magnitudes affects the bins with highest values of $S$, whereas saturation depopulates the low $S$ bins at the right. The presence of a significant population of objects in Lupus 1 and 3 is obvious as a local peak in the valley left between the $S$ regions dominated respectively by field main sequence and background giant contamination. It is also noticeable that only a relatively minor fraction of the objects contributed to this peak is composed of previously known members.

located within a range of values of $S$ that is not populated either by field cool dwarfs or by background cool giants. The location of this range, which depends on the distance, would move into the background-dominated part for aggregates closer than $\sim 100 \mathrm{pc}$, and is masked by the field stars for regions more distant than $\sim 300 \mathrm{pc}$.

To reveal this effect in our surveyed region, Fig. 5 shows the histogram of values of $S$ for four different ranges of temperature for each of the three surveyed clouds. The expected contribution to the source number counts by field dwarfs and giants in the same temperature range is given as well. Such contribution varies as a function of the area covered in each cloud and its position on the sky, mainly through its proximity to the galactic plane, which determines the level of background contamination.
At large values of $S$ (to the left in the plots) the field dwarf population dominates, with numbers being in rough agreement with the expectations yielded by the local initial mass function. The latter has been modeled using the log-normal forms given by Chabrier (2003, 2005), whose differences, according to Chabrier (2005), largely reflect the uncertainties in the present determinations. We find that Chabrier (2003) provides a closer match to the high- $S$ part of our histograms. The agreement is particularly good in the $3100<T_{\text {eff }}(\mathrm{K})<$ 3400 range and to some extent also below $\sim 2800 \mathrm{~K}$, where the number of stars that we observe is close to the model predictions for all three clouds. The discrepancies are larger for the $3400<T_{\text {eff }}(\mathrm{K})<3700$ interval, where our number counts fall significantly below the predicted ones, and for the 
$2800<T_{\text {eff }}(\mathrm{K})<3100$ interval, where the opposite is seen. Possible causes for this discrepancy are deviations between the actual initial mass function and its adopted form, inaccuracies in the adopted mass-temperature relationship for field dwarfs (for which we adopted the $5 \mathrm{Gyr}$ isochrone of Baraffe et al. 1998), or systematic effects in the temperature derived for evolved field objects of higher surface gravity when using the $\log g=4.0$ synthetic spectra that are more appropriate for the young population of the Lupus clouds. An additional cause for the lower-thanexpected number of field stars observed in the $3400<T_{\text {eff }}(\mathrm{K})<$ 3700 interval comes from the fact that the highest values of $S$ covered by our histograms correspond to main sequence stars located beyond the Lupus clouds, and thus subjected to obscuration by them. Although such effect is no doubt present in our data, the relatively small fraction of the field observed toward each cloud that is covered by highly obscuring dust leads us to conclude that this cause alone cannot explain the observed discrepancy.

It is more difficult to compare our results with the contamination expected from background cool giants since such contamination, which becomes important at the lowest values of $S$, corresponds mostly to stars that are saturated in our images, unless they are sufficiently obscured by dust for their magnitudes to fall below our saturation limits. The fact that our number counts of unsaturated stars fall in all cases below the predicted number of background cool stars is consistent with this. The effect of galactic latitude on the expected contamination by cool giants is rather dramatic, as discussed in Sect. 3.2. Very few cool giants are expected in the direction of Lupus 1 as compared to Lupus 3 and even more with Lupus 4.

The plots in Fig. 5 show the evidence of substantial young populations in Lupus 1 and especially in Lupus 3, and only a trace of such a population in Lupus 4. Such populations appear as an excess in the histograms of detected objects with respect to the sum of the contamination due to field dwarfs and background giants within a range of values of $S$. Our histograms also indicate as a function of $S$ the number of previously known members of the clouds within the considered temperature interval. In this way it is also clearly seen that both Lupus 1 and Lupus 3 contain significant amounts of thus far unrecognized cool members. It should be noted that the known members contributing to the histograms plotted in Fig. 5 are only those that we detect in at least $I_{\mathrm{C}}$ and $z_{\mathrm{WFI}}$ and for which we therefore derive $S$ and $T_{\text {eff }}$ in exactly the same way as we do for all other stars in our images. Therefore, such contribution to the histograms does not include known embedded members that are too obscured to be detected in those bands or, at the brighter end, members within the relevant range of temperatures that are saturated in either of those bands.

Although the approach to recognize the existence of the populations associated with the clouds is in principle statistical, the fact that such populations occur in regions of the $S$ histogram devoid of contamination allows us to produce lists of individual new candidate members whose intrinsic parameters are derived with the procedure outlined in Sect. 3.1. We note that the new members that we report here are unlikely to possess significant excess emission in the $0.6 \mu \mathrm{m}-2.2 \mu \mathrm{m}$ range or veiling at short wavelengths, since the warm disks and accretion that produce them would most likely have been detected in previous studies. This increases our confidence in the derivation of their properties, as their spectral energy distributions are expected to be free from some of the causes that distort the fits to the objects listed in Table 4. Indeed, good quality fits (residual $<0.2 \mathrm{mag} \mathrm{rms}$ ) are obtained for virtually all the new candidate members.

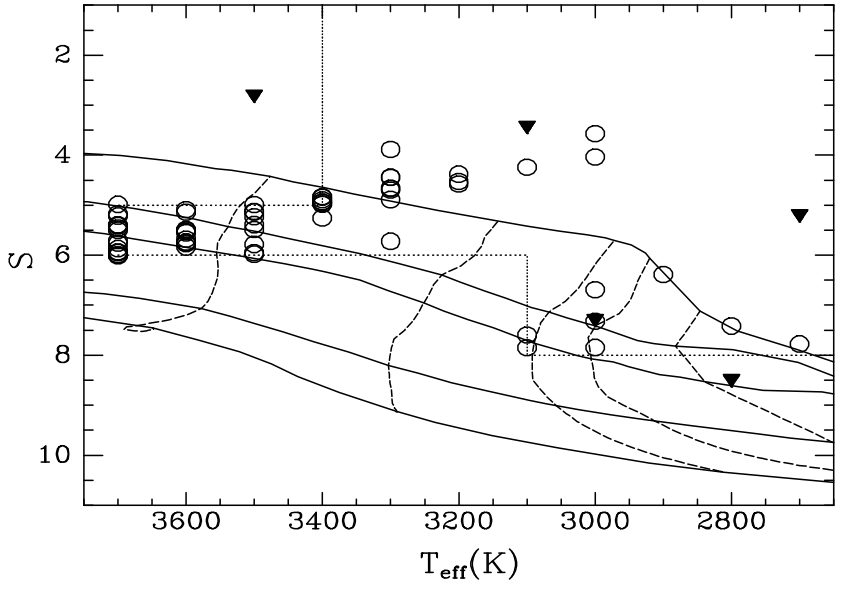

Fig. 6. Diagram showing the positions of both known and new candidate members of Lupus 1 based on their presence in regions of the $T_{\text {eff }}-S$ diagram expected to be devoid of contamination by field main sequence stars and background giants. Filled triangles indicate previously known members for which we derived $T_{\text {eff }}$ and $S$ using Eqs. (4), and open circles are newly identified objects with their parameters calculated in the same way. The area delimited by the dotted lines is the region adopted as contamination-free based on starcount models and on the actual histograms of $S$ values for sources within the relevant temperature range. The solid curves are the isochrones computed by Baraffe et al. (1998) (masses of $0.075 M_{\odot}$ and above) and Chabrier et al. (2000) (substellar masses below $0.075 M_{\odot}$ ) for ages $1,5,10,50$, and $5000 \mathrm{Myr}$, from top to bottom. The dashed lines depict the evolutionary path followed by stars of masses $0.2,0.1,0.075,0.05$, and $0.02 M_{\odot}$, from left to right.

\subsection{Lupus 1}

The histogram of cool stars with $3400<T_{\text {eff }}(\mathrm{K})<3700$ of Lupus 1 peaks at $5<S<6$, just where contamination by field dwarfs and giants is expected to reach a minimum, thus clearly revealing the signature of the population associated with the cloud. None of the stars contributing to this bin has been previously recognized as a member of Lupus 1 . In this range of temperatures such values of $S$ correspond to members of the cloud population with typical ages between 2 and more than $10 \mathrm{Myr}$, the actual limits depending on the precise temperature. Younger objects with $S<5$ are generally above our saturation limit, which may explain why the peak moves to $4<S<5$ in the next temperature interval, $3100<T_{\text {eff }}(\mathrm{K})<3400$. The presence of the cool Lupus 1 members with $S>6$ and $T_{\text {eff }}>3400 \mathrm{~K}$ is difficult to ascertain from our observations, since foreground contamination dominates in this range.

Virtually no contamination by non-members is expected for $S<8$ below $T_{\text {eff }} \simeq 3100 \mathrm{~K}$. However, we find 15 objects in this range, only three of which were previously known and listed in Table 4. We also consider all the stars with $S<6$ in the $3100<T_{\text {eff }}(\mathrm{K})<3400$ range, and the stars with $5<S<6$ in the $3400<T_{\text {eff }}(\mathrm{K})<3700$ range, as candidate new members, although these latter are likely to include a few field stars. None of these 53 additional stars had been previously identified as a possible member. We thus propose a census of 65 new members of Lupus 1 with $T<3700 \mathrm{~K}$, which we list in Table 9. The actual census of previously unidentified members most probably extends beyond the $S$ ranges that we chose here on the basis of the weak contamination.

The location of these new members in the $T_{\text {eff }}-S$ diagram is plotted in Fig. 6, together with the isochrones, evolutionary tracks, and the limiting values of $S$ described above for each temperature range. The narrow limits of $S$ chosen for 


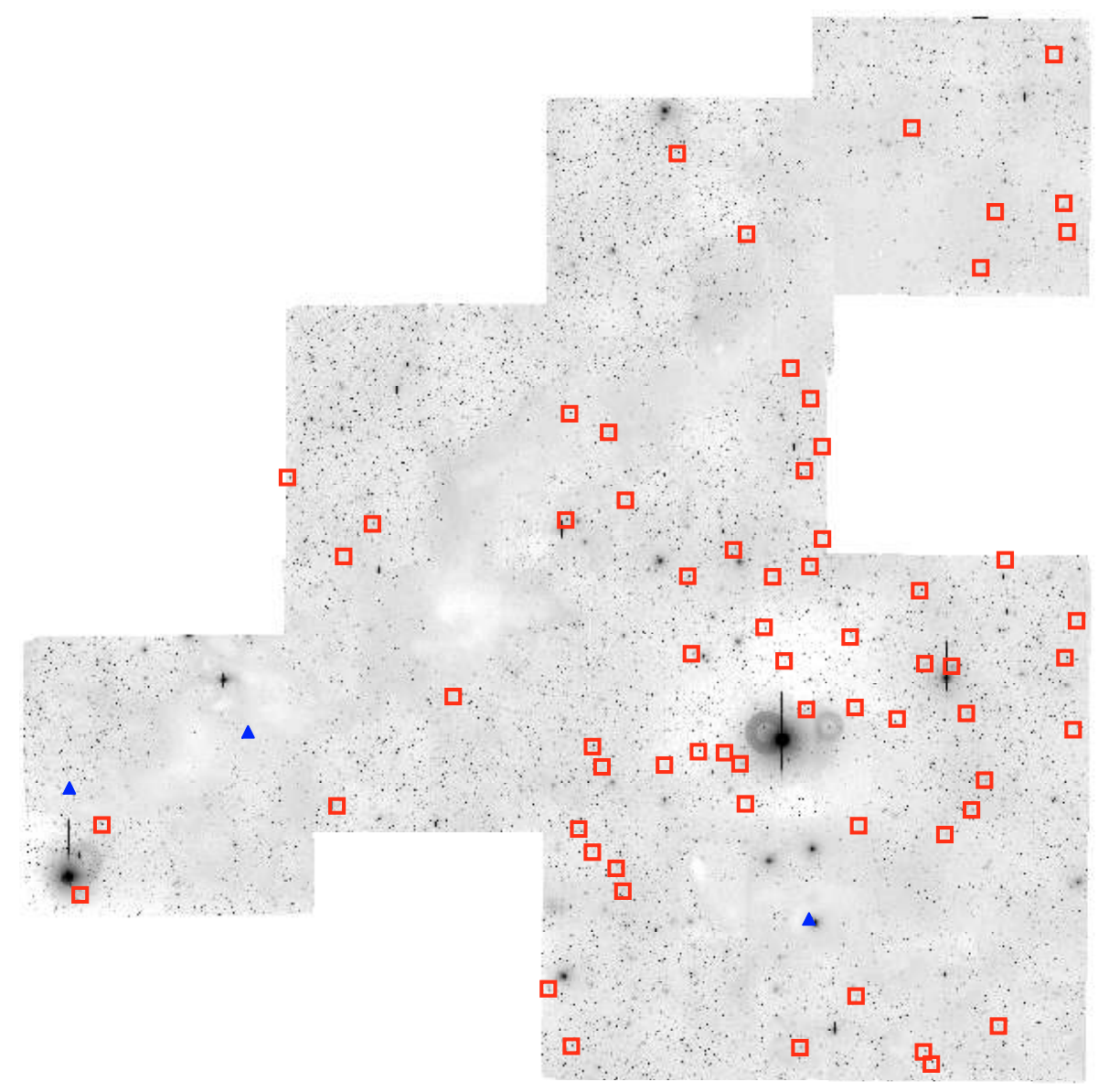

Fig. 7. Distribution of previously known (filled triangles) and newly identified (open squares) members of Lupus 1, plotted on a $R$-band mosaic of the region constructed from our WFI observations. Note that in order to produce a homogeneous comparison between the distributions of known and new members, only the known members lying within the limits of $S$ defining the contamination-free region of the $T_{\text {eff }}-S$ diagram are plotted. Therefore, some of the known members plotted in the corresponding $T_{\text {eff }}-S$ diagram do not appear here. The field measures $129^{\prime} \times 129^{\prime}$ and is centered on the position $\alpha(2000)=15^{\mathrm{h}} 42^{\mathrm{m}} 00^{\mathrm{s}}, \delta(2000)=-34^{\circ} 03^{\prime}$. North is at the top and East to the left.

$T_{\text {eff }}>3400 \mathrm{~K}$ imply that we sample a narrow range of ages at such temperatures, probably leaving aside the youngest members as they lie in an area where contamination by background stars becomes substantial. Evidence for such a younger population, with ages below $2 \mathrm{Myr}$, appears at lower temperatures where no lower limit in $S$ is needed due to the expected absence of background contamination. In addition, the recently identified member SSTc2dJ153803.1-331358 (Merín et al. 2008), with $T_{\text {eff }}=3500 \mathrm{~K}$, also appears in the zone corresponding to ages below $1 \mathrm{Myr}$.

Only one of our proposed new members, J154433.9-335254, displays signatures of warm circumstellar dust detectable by Spitzer, which appears as a moderate excess at $24 \mu \mathrm{m}$ and perhaps a slight excess at $8 \mu \mathrm{m}$ indicating the absence of dust warmer than a few hundred K. Although most of the other new Lupus 1 members have been detected by Spitzer, all of them have mid-infrared spectral energy distributions typical of pure photospheres.

The $S<8$ limit imposed to avoid contamination by field stars prevent us from identifying cool stars $\left(T_{\text {eff }}<3100 \mathrm{~K}\right)$ with ages above 5-10 Myr. However, the younger end of this range is accessible at temperatures above $3400 \mathrm{~K}$ as noted before. The abundance of new members found in this range leads us to suspect that star formation in Lupus 1 started well over $10 \mathrm{Myr}$ ago, although the identification at present of the oldest members is problematic since most of them may not be accreting significantly or have detectable disk emission anymore.

The distribution on the sky of the new members that we identify is plotted in Fig. 7. Together with the new members we also plotted the position of those known members that are selected using the same criteria. The known members plotted in those figures are therefore not the complete list, since it excludes both objects for which we cannot produce a fit (such as those too deeply embedded to be detected in the visible or those saturating in either $I_{\mathrm{C}}$ or $z_{\mathrm{WFI}}$ ) and objects falling outside the ranges of $S$ used to select samples uncontaminated by field and background stars. We preferred to proceed in this way so that an unbiased comparison can be established between the distributions of known and new members. The same procedure has been followed in the plots of the distribution of members of Lupus 3 and 4, discussed in the next Sections. The number of members thus excluded from the plots is 10 in Lupus 1, 72 in Lupus 3, and 10 in Lupus 4.

The few previously known members of the Lupus 1 clouds (including those not plotted in Fig. 7) tend to concentrate toward the two main masses of dark nebulosity covered by our observations, TGU H2079 (Dobashi et al. 2005), the elongated cloud running from SE to NW) and TGU H2072 (the more round cloud on the SW region of the field). In contrast, the new members show a concentrated distribution roughly centered in the area between both dark clouds, with no particular association with either of them. 


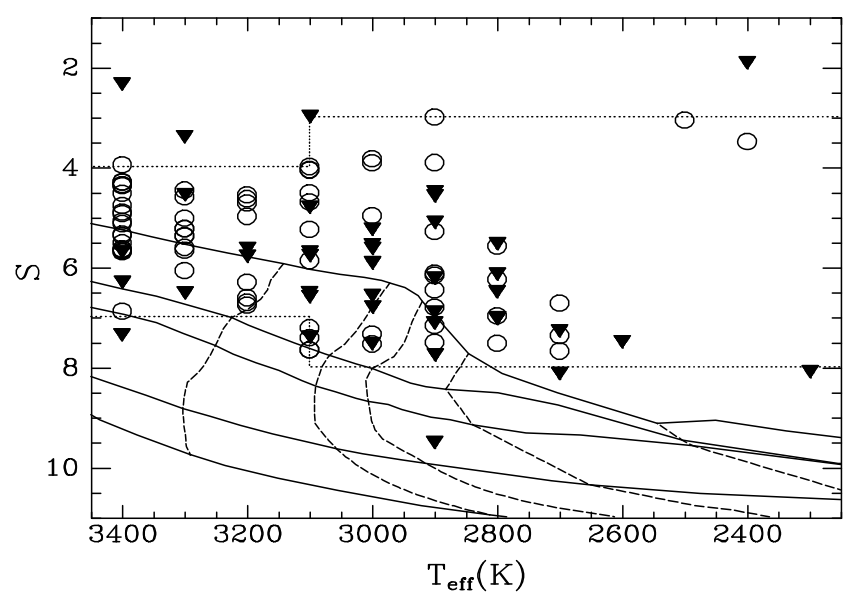

Fig. 8. Same as Fig. 6 for Lupus 3. Note the different limits in the temperature axis, since stars with temperatures above $T_{\text {eff }}=3400 \mathrm{~K}$ are now excluded due to the stronger degree of contamination.

\subsection{Lupus 3}

Qualitatively, the histograms of $S$ values in Lupus 3 are similar to those obtained for Lupus 1 . However, the lower galactic latitude of Lupus 3 leads to a dramatic increase in the surface density of background cool giants expected to appear in our surveyed field. Thus, although the histogram of $S$ values for the range $3400<T_{\text {eff }}(\mathrm{K})<3700$ clearly shows an excess of sources associated with the Lupus 3 young stellar population and a peak at the same position as in Lupus 1, all the bins in that temperature range are expected to contain significant contamination by either foreground or background sources. We thus preferred to leave the $3400<T_{\text {eff }}(\mathrm{K})<3700$ range outside our discussion of Lupus 3, since the likelihood for any star in it to be a non-member is high. Restricting ourselves to the $5<S<7$ range where the excess of sources over the contamination prediction stands out, we estimate the existence of roughly 60 thus far unidentified members of the Lupus 3 aggregate.

The signature of the Lupus 3 population is much clearer at lower temperatures. The excess of sources over the predicted numbers of field dwarfs and giants in the $4<S<7$ range for $3100<T_{\text {eff }}(\mathrm{K})<3400$ is more than 40 , out of which only 6 are known. The signature is also clear for $3<S<8$ at $T_{\text {eff }}<3100$, where we find 58 objects, 31 of them previously unrecognized. The 72 new candidate members that we find with $T_{\text {eff }}<3400 \mathrm{~K}$ within these boundaries are listed in Table 9; we estimate the number of contaminants in this range to be no more than 10. Although the number of new candidates that we identify in Lupus 3 is similar to that in Lupus 1, we note that most of the candidates in the latter cloud have $T_{\text {eff }}>3400 \mathrm{~K}$, which we exclude from our Lupus 3 census.

Unlike in the case of Lupus 1 discussed above, we need now to use a lower limit to the values of $S$ for candidate members due to the significant expectation of background contamination. We note in this respect that the two coolest stars in our list of candidate members, J160523.5-391849 ( $S=3.1$ ) and $\mathrm{J} 161353.9-380850(S=3.5)$, although formally within the boundaries of our member selection criterion, are close to the values of $S \simeq 3.0$ where background contamination becomes significant. We therefore consider them as likely background stars, and we note it accordingly in Table 9. Another object with even lower $S$, outside our boundary, is SSTc2dJ161001.1385401, which Merín et al. (2008) classify as a heavily obscured early-type member of Lupus 3. The fit that we obtain with a low temperature leads us to consider it instead as another likely cool giant as noted in Table 4, or perhaps a AGB star, which may explain its mid-infrared excess.

An intriguing difference between the populations identified in Lupus 1 and 3 is the considerably larger fraction of members that are already known from previous studies, which is readily apparent from Fig. 5. At first sight the difference might be attributed to the greater attention that Lupus 3 has traditionally received, with more studies aimed at revealing new members. However, the comprehensive surveys carried out with Spitzer dealt with both regions in the same manner, with very similar sensitivity levels and using identical criteria for the detection of young stellar object candidates, yet they detected many more in Lupus 3 than in Lupus 1; see Table 4 of Merín et al. (2008). It thus seems very unlikely that the difference is largely due to the more detailed study received by Lupus 3 in the past. Instead, the fraction of Lupus 3 members displaying characteristic midinfrared excess is truly higher than in Lupus 1.

A small group of 7 new Lupus 3 members display midinfrared excess as well. Based on the position in the [3.6]-[4.5], [5.8]-[8.0] diagram we identify three objects with excesses characteristics of young stellar objects. The source J160836.2392302 displays clear excess emission starting at $4.5 \mu \mathrm{m}$, and is also detected at the MIPS $70 \mu \mathrm{m}$ band. According to the classification criteria defined by the extensive disk simulation grid of Robitaille et al. (2006) it belongs to Stage I, corresponding to an object surrounded by an infalling envelope and possibly a disk. The other two excess sources in this diagram are J160800.2390259 and J160822.8-390058, lying in the area Stage II corresponding to objects surrounded by optically thick disks. The use of similar criteria in the [3.6]-[5.8], [8.0]-[24] diagram now places J160836.2-392302 in the Stage II area and confirms the classification of J160800.2-390259 within this same stage. The use of those two colors also shows the infrared excess displayed by two additional sources, J160804.8-390449 and J160831.6384729 , which shows clearly only at $24 \mu \mathrm{m}$. Both sources are near the dividing line between Stages II and III, marking the transition from thick to thin disks. Also J160822.8-390058, previously classified as Stage II and whose mid-infrared excess appears longwards of $5.8 \mu \mathrm{m}$, lies close to this boundary. Finally two other sources, J160836.4-393119 and J160826.8-384101, show clear excess in MIPS observations at $24 \mu \mathrm{m}$. Unfortunately, they lie in an area that was not observed by IRAC at shorter wavelengths.

We show the distribution of the new members of Lupus 3 in the $T_{\text {eff }}$ vs. $S$ diagram in Fig. 8, and their spatial distribution in Fig. 9, together with the distribution of those previously known members that are selected according the same criteria. Among the new members a similar degree of concentration as in Lupus 1 is observed, which in this case form a distribution roughly centered on the dense star-forming cloud TGU H-2084 hosting most of the known members and the Herbig Ae/Be stars HR 5999/6000. Although the dark cloud lies near the center of the distribution very few new members appear projected on it, in stark difference with the distribution of the known members. The fact that the known members were selected according to the same criteria argues against the difference between both distributions being due to extinction.

\subsection{Lupus 4}

The histogram of stars detected as a function of $S$ for Lupus 4 differs markedly from those of Lupus 1 and 3 in all the temperature bins in which Fig. 5 is organized. The source counts 


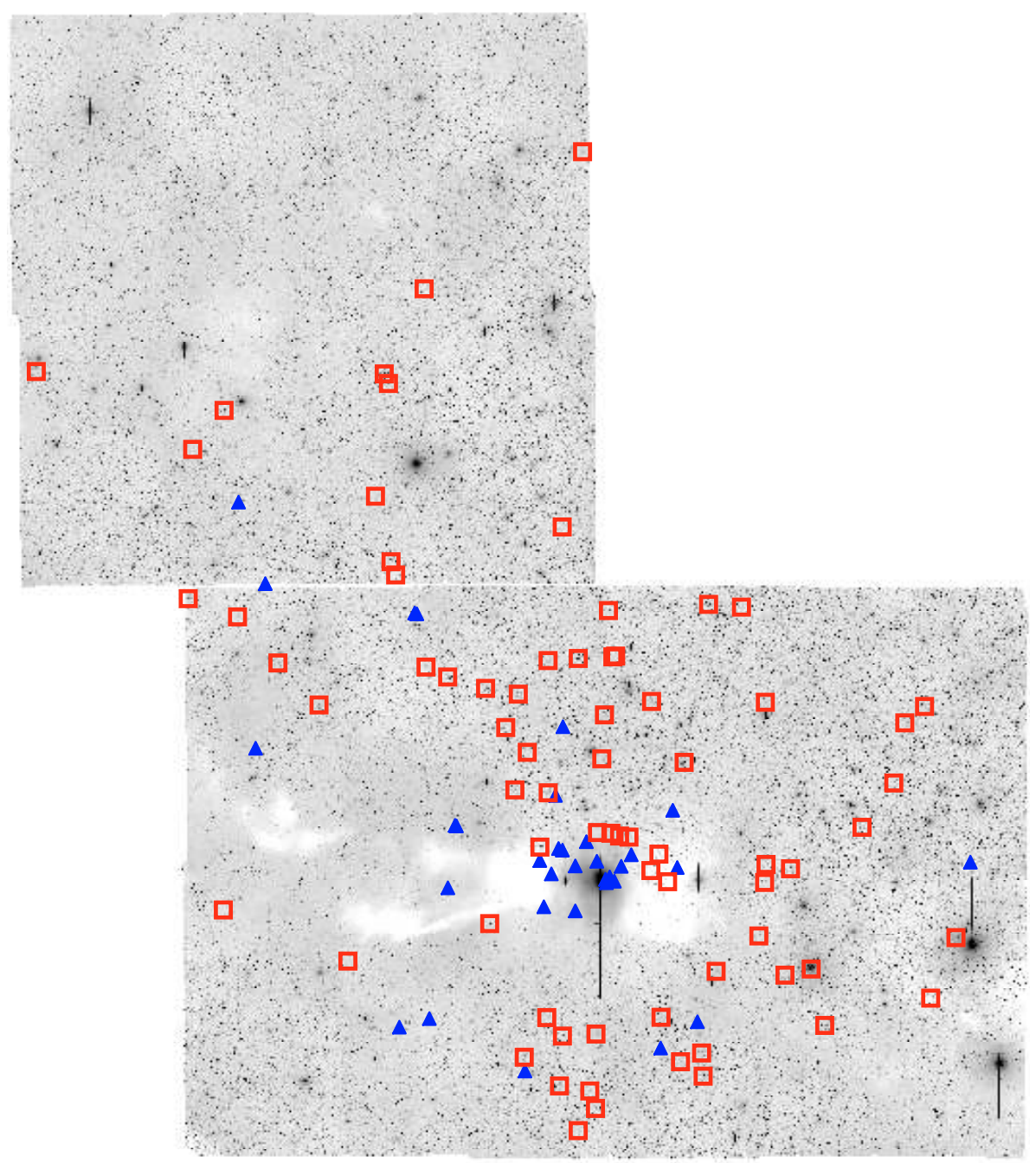

Fig. 9. Same as Fig. 7 for Lupus 3. The field is in this case $112^{\prime} \times 126^{\prime}$ in size and is centered on $\alpha(2000)=16^{\mathrm{h}} 09^{\mathrm{m}} 20^{\mathrm{s}}, \delta(2000)=-38^{\circ} 33^{\prime}$.

in this cloud are in general in good consistency with the contribution from the field dwarfs and giants alone, and the trace of a young population associated with it is only marginal. Given is galactic latitude $b=8^{\circ}$, the expected contamination by unrelated stars is very similar to that expected in the direction of Lupus 3, scaled down due to the smaller area covered by our observations of Lupus 4 . The peak at $5<S<6$ at $3400<T_{\text {eff }}(\mathrm{K})<3700$ can now be accounted for by background early $\mathrm{M}$ giants alone. We only identify 6 apparent members of the cloud with $T_{\text {eff }}<3100 \mathrm{~K}$, one of them previously known. The other 5 are listed in Table 9 . Three additional members may be present in the $3100<T_{\text {eff }}(\mathrm{K})<3400$ range with $4<S<5$, although background giants are expected to contribute to this bin of the histogram and we do not consider this excess to be sufficiently significant. We only identify two stars in the virtually uncontaminated $5<S<7$ range, one of which is a known member. Only one of the newly identified candidate members of Lupus 4, J160019.2-415635, has its mid-infrared flux measured by Spitzer, without indication of significant excess flux through the $3.6 \mu \mathrm{m}-24 \mu \mathrm{m}$ range. The positions of all these objects in the $T_{\text {eff }}$ vs. $S$ diagram is shown in Fig. 10.

The spatial distribution of the few objects identified in Lupus 4, shown in Fig. 11 does not show any hints of concentration toward the central cloud, TGU H-2063, and none of them appears projected on it. In this respect, it is worth noting

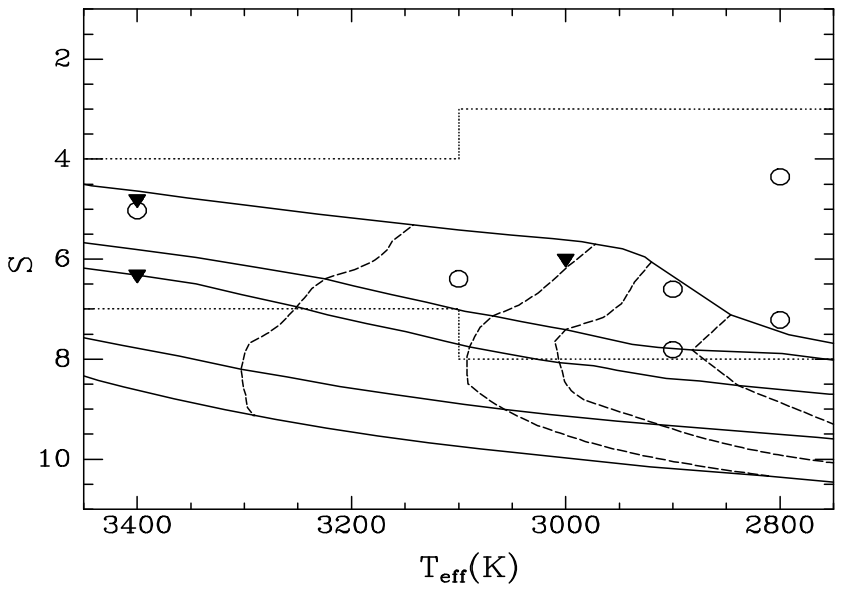

Fig. 10. Same as Fig. 6 for Lupus 4. Like in Lupus 1, the limits of the temperature axis are conveniently adjusted.

the striking difference between Lupus 1 and 4. Previous to the present study, and even after the surveys carried out by Spitzer, the number of known members in Lupus 1 and 4 was very similar (see Table 9 in Merín et al. 2008). However, whereas our results add 64 objects to the 17 known in Lupus 1, the increase is a modest 6 to the 18 known in Lupus 4, and the true members 


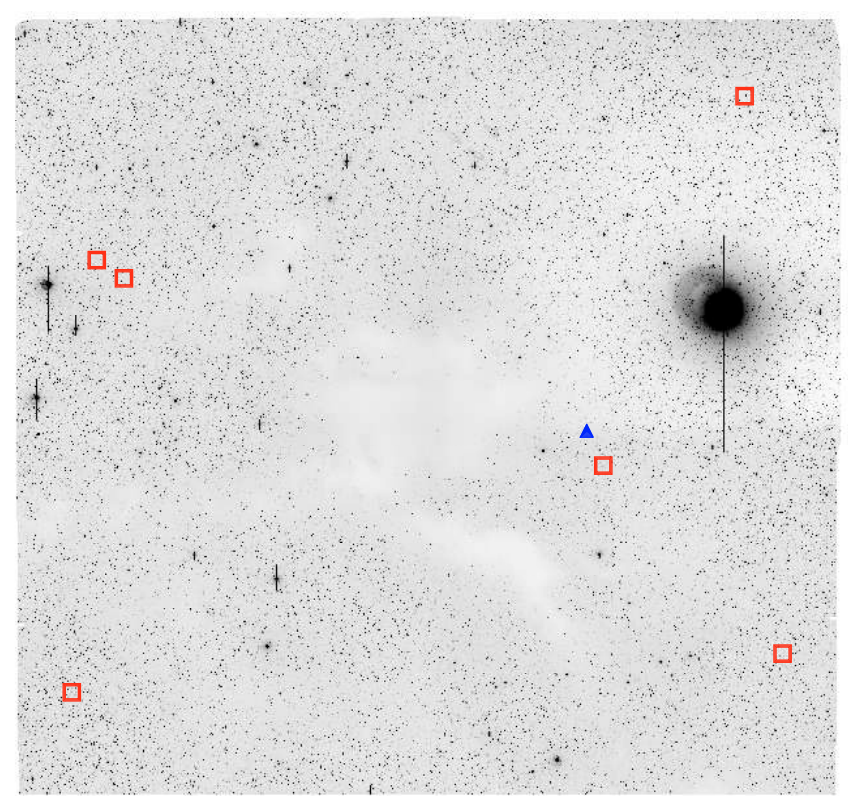

Fig. 11. Same as Fig. 7 for Lupus 4 . The field is in this case $62^{\prime} \times 64^{\prime}$ in size and is centered on $\alpha(2000)=16^{\mathrm{h}} 01^{\mathrm{m}} 32^{\mathrm{s}}, \delta(2000)=-41^{\circ} 54^{\prime}$.

in this latter case may be even less as noted in Sect. 6. In this regard, our results for Lupus 4 closely resemble those of Spezzi et al. (2007), who found only a small number of additional candidate members of the Chamaeleon II clouds beside those already identified through the emission of their circumstellar envelopes. Both the selection criteria of known members of Chamaeleon II, mainly based on Spitzer colors, and the method used to identify new candidates from broad-band imaging were very similar to those used in the present paper.

\section{Discussion}

The discovery of such a numerous population of new members of the Lupus 1 and 3 clouds is in itself an intriguing result, since it reveals that much of the stellar content of these clouds, and even most of it in the case of Lupus 1, has remained unidentified to date. The examination of the bulk properties of the new population and its comparison with those of the known members leads to some surprising results. Since we restrict our identification of new members to regions in the $T_{\text {eff }}-S$ diagram devoid of contamination by other cool stars, with a subsequent restriction on the age range from which the new members are drawn, we have limited information on the age distribution of the Lupus stellar population. In particular we cannot tell from our results if the total population of non-identified members reaches to older ages than that of known members, and we cannot use the newly identified members to reconstruct the history of star formation in Lupus, although the hotter stars of Lupus 1 hint at significant star-forming activity over $10 \mathrm{Myr}$ ago. For similar reasons there is little we can conclude about the stellar mass function in the Lupus clouds. Nevertheless, a comparison between the positions in the $T_{\text {eff }}$ vs. $S$ diagram of previously known and new candidate members presented in Figs. 6 and 8 does not show any significant differences between both groups in either age or mass distribution.

Our results show that the large population of newly discovered candidate members found in Lupus 1 and 3 contains numerous members with ages below 5 Myr or even 1 Myr and with no detectable signatures of disks or accretion, in the same range of ages covered by members that do display those signatures. The lack of noticeable infrared excess at $24 \mu \mathrm{m}$ for most of the new members implies the clearing of the inner parts of disks within at least the inner $1 \mathrm{AU}$ from the central object. The large number of new members without such disk signatures appears to be at variance with the research on the disk-bearing fraction of young stellar populations, which indicates a typical duration of 2 Myr for the optically thick disk phase (see Evans et al. 2008 for a summary of lifetime estimates for the different evolutionary phases, as derived by a large number of studies in different starforming regions). Haisch et al. (2001) show that about half of the stars in clusters are able to retain inner disks producing excess at $3.4 \mu \mathrm{m}$ in the first $3 \mathrm{Myr}$. The fraction seems to be similar or even higher at very low masses (Jayawardhana et al. 2003), including the giant-planet-mass regime (Scholz \& Jayawardhana 2008). On the other hand, low mass stars and brown dwarfs have been shown to frequently retain their disks for ages as old as $\sim 10 \mathrm{Myr}$ (Riaz \& Gizis 2008; Uchida et al. 2004), as well as to maintain a low accretion rate (Lawson et al. 2004) at those ages. The existence of coeval members with differing disk and accretion characteristics, such as classical T Tauri stars, weak-line T Tauri stars, and transition objects has been observed in other clusters, including the 9 Myr-old $\eta$ Cha (Lyo et al. 2003; Lawson et al. 2004), indicating that different paths towards disk dissipation, acting on different timescales, must exist. Additional evidence of the complexity of the disk dissipation process is presented by Merín et al. (2008), on the basis of basic parameters of the midinfrared spectral energy distribution that characterize the sizes of the inner clearings and the optical thickness and flaring of disks. The broad range of values and poor correlations between such a parameters indicate a wide variety of disk inner holes and geometries during the dissipation process. On the other hand, an examination of the mid-infrared excesses of weak T Tauri stars (WTTS) by Cieza et al. (2007) has shown that WTTS as young as 1 Myr exist with no trace of circumstellar dust, in consonance with our results.

We examined the fraction of wide binaries among the new population by searching for companions within a radius of $10^{\prime \prime}$ from each new candidate member (projected distance $1500 \mathrm{AU}$ at the distance of Lupus 1 and 4, and $2000 \mathrm{AU}$ at that of Lupus 3), and having $T_{\text {eff }}$ and $S$ compatible with membership in the corresponding cloud. Although most of the new candidate members have sources within that angular distance, in nearly all cases where their spectral energy distribution can be fitted the possible companions have $S$ values placing them below the main sequence, indicating that they are unrelated foreground stars instead. We only identify two possible binaries, both in Lupus 1, which are tabulated in Table 8. The first one, $\mathrm{J} 153815.5-350342 \mathrm{~B}$, is a possible brown dwarf companion with a derived age similar to that of the primary, lying slightly above the $S$-cutoff that we set at its temperature, $T_{\text {eff }}=2900 \mathrm{~K}$. The second companion candidate, 154018.5-342614B, is more doubtful, as we obtain a temperature slightly higher than that of its primary but a lower luminosity, which if taken at face value would imply an older age. However, the uncertainties in the derivation of the temperatures using our methods do not allow us to rule out the possibility that it forms a physical pair with its primary. While this hints at a low binary frequency among the new members, we must stress that our criteria for the identification of binaries is rather restrictive. On the one hand, we are unable to verify as likely companions low luminosity sources fainter than the detection limits of 2MASS, as a reliable fit cannot be derived from $R_{\mathrm{C}} I_{\mathrm{C}} z_{\mathrm{WFI}}$ photometry alone. On the other hand, some of our new candidate members may be companions 
Table 8. Possible binaries.

\begin{tabular}{lccccccc}
\hline \hline Object & $T_{\text {eff }}(\mathrm{K})$ & Sep. $\left(^{\prime \prime}\right)$ & $K_{S}$ & $L\left(L_{\odot}\right)$ & $A_{V}(\mathrm{mag})$ & $M\left(M_{\odot}\right)$ & age $(\mathrm{Myr})$ \\
\hline $153815.5-350342 \mathrm{~B}$ & 2900 & 4.3 & 13.61 & 0.005 & 1.6 & 0.05 & 10 \\
$154018.5-342614 \mathrm{~B}$ & 3500 & 6.0 & 10.16 & 0.072 & 0.3 & 0.45 & 18 \\
\hline
\end{tabular}

to brighter stars that appear saturated in some of our images. A dedicated investigation is thus necessary to derive the true incidence of binarity in our sample, and its possible relationship with its lack of disk signatures.

Could the new population be actually related to the Gould Belt, rather than to the star-forming Lupus clouds? Given the radial extent of the Gould Belt (see Sect. 3.2) such explanation would remove the problem of having to account for the quick dispersal of disks, since the position of these objects in the same $T_{\text {eff }}-S$ diagram would then be due to their closer distance to us rather than to a young age. However, our results provide several arguments against that explanation. In the first place, the new members of both Lupus 1 and Lupus 3 clearly show a concentrated spatial distribution, rather than a random scatter as would be expected from the Gould Belt population on the angular scale covered by our observations (see for instance Fig. 3 of Krautter et al. 1997, see also Wichmann et al. 1997, and Sterzik et al. 1995 for similar observations in the Orion region). Secondly, the surface density of our objects is much smaller in Lupus 4, which is close to Lupus 3 on the sky and should be affected by Gould Belt members to a very similar degree. A third argument is given by the $T_{\text {eff }}-S$ diagrams of Lupus 1 and, more notably, of Lupus 3, where no members, either new or already known, are found above a temperature-dependent value of $S$, which probably marks the location of the stellar birthline (Stahler 1983) in Lupus. Unless the inner edge of the Gould Belt casually happens to lie at the right distance, there is no reason why its members should not occupy the part of the diagram above this line.

Of course, the arguments above do not exclude the possibility that some of the new members that we identify do belong to a foreground population associated with the Gould Belt. As noted in Table 9, some of them were identified by Krautter et al. (1997) thanks to their X-ray emission as weak-line T Tauri stars and possible members of that population. The small number of new candidate members that we detect toward Lupus 4 and their lack of noticeable clustering toward the cloud suggest that perhaps all the objects that we tentatively identify as new members of Lupus 4 may belong instead to the foreground Gould Belt population. However, there is no doubt that this is at most a minor contributor to the number of new members that we find toward Lupus 1 and 3.

\subsection{The origin of the new members}

A surprising result of our study is the different spatial distribution between the newly discovered objects often without disk signatures, mostly located outside the main clouds, and those that preserve their youth signposts. The locations of the latter are very closely correlated to the densest concentrations of molecular gas and dust, as noted by Merín et al. (2008). A difference in ages could in principle be an obvious cause for the spatial segregation between both populations, reflected also on their different disk properties. In this picture, the oldest objects would have had time to slowly drift away from the parental clouds while their disks evolved toward dissipation. However, as noted above we find no evidence of the age differences between both populations that would be required by such explanation. We cannot rule out that such differences might be revealed by future studies that will more accurately determine the intrinsic properties of the new candidate members, including their ages, but at the moment the available data provide no support for their existence.

It is very unlikely that the halo of new members around TGU-H2084, the main molecular condensation of Lupus 3, might be composed by objects formed in the cloud and then ejected shortly after their formation, losing the disk in the process. Taking as typical values for our new members an angular distance to the cloud of $0^{\circ} 5$ and a travel time of $\sim 0.5 \mathrm{Myr}$, the ejection velocities that are required are not very high, of order of $\sim 4 \mathrm{~km} \mathrm{~s}^{-1}$. However, the efficiency of the process of ejection involving stripping of the disk would need to be extremely high to account for the production of roughly 70 objects or more displaying such features, a number that is comparable to that of objects in the same age range that retain their disks. We thus regard this as a very unlikely explanation. Furthermore, it is doubtful that it may apply to Lupus 1 as well, since the concentration of new members without disk signatures does not appear to be centered on any of the observed clouds -and where, if correct, the ejection plus disk-stripping mechanism should have affected to most of the members to account for the ratio of diskless to disk-bearing stars.

Alternatively, all the objects may have formed near their present locations and their disks may have then followed an environment-dependent path, with objects embedded in the dense clouds being able to retain their inner disks and accretion rates for millions of years, whereas those forming outside the clouds lost them much more quickly. A reason for these different evolutionary paths may be related to the shielding provided to forming disks by the parental molecular cloud, which would protect them from being externally irradiated, whereas this would not be the case for exposed disks. The fast evaporation of disks by the ultraviolet radiation of nearby $\mathrm{O}$ stars is well known in the Trapezium cluster, where protoplanetary disks resolved by the Hubble Space Telescope appearing in silhouette against a bright background are often distorted due to the proximity to the hot Trapezium stars, in agreement with the results of numerical simulations (Richling \& Yorke 1998). Johnstone et al. (1998) and Bally et al. (1998) estimate mass loss rates due to disk photoevaporation as high as $10^{-7} M_{\odot} \mathrm{yr}^{-1}$, and disk lifetimes as short as $0.1 \mathrm{Myr}$ or less in these conditions. Further evidence is presented by Robberto et al. (2004), who derive mass accretion rates on low-mass stars in the Trapezium cluster. The typical accretion rates found are on the average one order of magnitude below those derived for other star-forming regions such as the Taurus clouds or adjacent regions in Orion itself, despite the similar distributions in mass and age. The authors attribute this difference to the fast exhaustion of the disk reservoirs for accretion as the O-type members of the Trapezium evaporate them. They also propose that the early suppression of accretion by the dissipation of the disk should result in a peculiar Initial Mass Function biased toward low mass members, as their 
further growth is prematurely terminated. The effects of external disk irradiation are nevertheless detectable only on the close surroundings of the Trapezium, on distances of $\sim 0.1 \mathrm{pc}$, which are over one order of magnitude smaller than the size of the region occupied by the new members of Lupus. Evidence for fast disk dissipation on a longer length scale has been reported in the Rosette nebula cluster, where two jets are detected emanating from stars otherwise lacking any signatures of the existence of a disk (Li \& Rector 2007). This is interpreted as the result of the early and fast transition from the classical to the weakline $\mathrm{T}$ Tauri phase triggered by the external erosion of the disk by nearby $\mathrm{O}$ stars, which leaves the jet as the only recognizable signpost of youth. In this case the distance between the jetdriving stars and the nearest massive stars is in the $2-3$ pc range, but the eroding radiation field is more intense by more than one order of magnitude than in the Trapezium, mainly because of the presence of very massive $\mathrm{O} 4 \mathrm{~V}(\mathrm{f})$ and $\mathrm{O} 5 \mathrm{~V}$ stars within that distance range.

In our case, the candidate sources of external irradiation may be the OB stars of the Scorpius-Centaurus OB association, one of whose groups, Upper Centaurus-Lupus, is adjacent to the Lupus clouds (Blaauw 1991). However, no O-type stars are found at present within few parsecs of either Lupus 1 or Lupus 3 (de Zeeuw et al. 1999), thus rendering such explanation highly unlikely. With the disk mass loss rate $\dot{M}$ decreasing with increasing distance $d$ to the ionizing source as $\dot{M} \propto d^{-1.1}$ (Richling \& Yorke 1998), the mechanism quickly loses efficiency in the absence of very nearby massive stars.

Preibisch \& Zinnecker (1999) suggested that star formation in the Lupus clouds may have been triggered by a supernova explosion about 5 Myr ago in the Upper Scorpius OB association. At a distance of several parsecs, the shock wave driven by the explosion in the surrounding interstellar medium would have slowed down by collecting intervening material, most probably being in the radiative shock phase (Chevalier 1977) by the time it reached the Lupus clouds. Slow shocks can trigger the outside-in collapse of preexisting clouds, as described by Boss (1995), via the thermal pressure by the post-shock material. This results in the fast collapse of the cloud giving rise to a high accretion rate (up to $10^{-4} M_{\odot} \mathrm{yr}^{-1}$ ) on the central object, which rapidly declines in the absence of a massive reservoir of gas. This provides another possible explanation for the existence of an off-cloud population, which may have formed as a result of the direct exposure of isolated small molecular cores to one or several successive shock waves from the neighboring OB association. The outside-in character of the collapse, as opposed to the inside-out collapse of the cores embedded in the more quiescent environment of the larger molecular clouds, may explain the very different disk properties that we identify. With respect to the previous explanation based on the external irradiation of disks, the advantage of the supernova-triggered star formation scenario is that it does not require the presence of very nearby massive stars, which renders it more plausible in the scenario of Lupus 1 and 3. The absence of this population in Lupus 4 (and probably also in other clouds, such as Chamaeleon II; Spezzi et al. 2007) may then be simply due to the lack of nearby supernovae having provided this triggering mechanism.

\section{Conclusions}

We have presented the results of a wide survey of the Lupus 1, 3, and 4 clouds which, when combined with 2MASS photometry, provides a broad wavelength coverage well suited for the determination of temperatures of cool stars and their line-of-sight extinctions by means of fits to synthetic spectra. We estimate in this way the temperatures of over 150000 stars in the directions of the Lupus clouds, including many previously known members with temperatures independently derived from their spectral types, and conclude that our method provides a generally good fit to the temperature, except when veiling, strong emission lines, variability, or nearby companions are present.

We introduce a parameter $S$ related to the ratio of the distance to the star and its radius. Using it, we show that members of the Lupus clouds at a given temperature and within a restricted set of ages that are in the range expected for a starforming region are characterized by values of $S$ virtually unreachable by non-members of similar temperature. We take advantage of this property, shared by other star-forming regions at similar distances from the Sun, to demonstrate the existence of an important population of members of Lupus 1 and 3 thus far undetected by previous surveys. This population is either much less significant or non-existing in Lupus 4.

The properties of this new population are puzzling in several respects. The ages of its components are young, in the range of the previously known members of the same regions, but their non-detection until now implies that they are surrounded by little or no circumstellar material within at least $\sim 1$ AU from the central object, as confirmed by the Spitzer photometry available for most of these objects. Furthermore, while the new populations discovered in Lupus 1 and 3 tend to be spatially concentrated (toward a region between the two main clouds forming the Lupus 1 complex, and toward the main cloud in Lupus 3), most of their members are located outside those clouds.

Taking together the absence of significant disk emission among the newly identified members and their preferential location away from the main molecular concentrations of their host complexes, we tentatively interpret our results in terms of the rapid disappearance (on timescales of less than $1 \mathrm{Myr}$ ) of the inner disks of stars formed outside the shielding environment of a molecular cloud. We consider it unlikely that the agent responsible for the fast dispersal of off-cloud stars in Lupus 1 and 3 may be the ultraviolet radiation of OB stars belonging to the Upper Centaurus-Lupus group of the Scorpius-Centaurus OB association, which is located adjacent to the Lupus clouds on the sky but whose individual members are too far away from the Lupus clouds. While evidence of disk ablation by external irradiation by $\mathrm{O}$ stars has been previously reported in the Trapezium and Rosette clusters, the applicability of this explanation to the Lupus clouds remains very doubtful due to the much less extreme radiation environment. We consider as more likely an alternative explanation by which the formation of the off-cloud population may have been caused by the passage of one or more shock fronts associated with evolved supernova remnants, triggering the outside-in collapse of preexisting cloudlets.

Regardless of its ultimate interpretation, the discovery of a substantial and even dominant population of thus far unnoticed members of one of our nearest star-forming regions stresses the important unknowns that still subsist in their observational characterization, despite the important advancements that recent surveys from the ground and from space have made possible.

Acknowledgements. We are pleased to thank the staff of La Silla Science Operations for the careful execution of our observing runs in Service Mode at the $2.2 \mathrm{~m}$ telescope, as well as to the ESO User Support Department for their assistance with the preparation of the observations. This paper benefitted from comments on the manuscript by Drs. Ewine van Dishoeck and Neal Evans, as well as by a careful review by the referee, Dr. Bruno Merín. F. C. is also thankful to Dr. Ewine van Dishoeck for first having invited him to become involved in the 'Cores to Disk' effort, and to the hospitality of the Vatican Observatory staff 
during the final stages of preparation of this paper. B.L.M. acknowledges the financial support of the ESO Director General Discretionary Fund and from the Spanish MICINN under the Consolider-Ingenio 2010 Program, through grant CSD2006-00070. This research has made use of the SIMBAD database operated at CDS, Strasbourg, France. It also makes use of data products from the Two Micron All Sky Survey, which is a joint project of the University of Massachusetts and the Infrared Processing and Analysis Center/California Institute of Technology, funded by the National Aeronautics and Space Administration and the National Science Foundation. This work is based in part on archival data obtained with the Spitzer Space Telescope, which is operated by the Jet Propulsion Laboratory, California Institute of Technology under a contract with NASA.

\section{References}

Allard, F., Hauschildt, P. H., \& Schwenke, D. 2000, ApJ, 540, 1005 Allen, P. R., Luhman, K. L., Myers, P. C., et al. 2007, ApJ, 655, 1095 Allers, K. N., Kessler-Silacci, J. E., Cieza, L. A., \& Jaffe, D. T. 2006, ApJ, 644, 364

Baade, D., Meisenheimer, K., \& Iwert, O. 1999, The Messenger 95, 15

Bally, J., Testi, L., Sargent, A., \& Carlstrom, J. 1998, AJ, 116, 854

Baraffe, I., Chabrier, G., Allard, F., \& Hauschildt, P. H. 1998, A\&A, 337, 403

Baraffe, I., Chabrier, G., Barman, T. S., Allard, F., \& Hauschildt, P. H. 2003, A\&A, 402, 701

Blaauw, A. 1991, in The Physics of Star Formation and Early Stellar Evolution, ed. C. J. Lada, \& N. D. Kylafis, NATO ASI Ser. C, 342

Bohlin, R. C., \& Gilliland, R. L. 2004, 127, 3508

Boss, A. P. 1995, ApJ, 439, 224

Burgasser, A. J. 2004, ApJS, 155, 207

Burgasser, A. J., Kirkpatrick, J. D., McGovern, M. R., et al. 2004, ApJ, 604, 827 Cambrésy, L. 1999, A\&A, 345, 965

Cardelli, J. A., Clayton, G. C., \& Mathis, J. S. 1989, ApJ, 345, 245

Chabrier, G. 2003, PASP, 115, 763

Chabrier, G. 2005, in The initial mass function 50 years later, ed. E. Corbelli, et al. (Springer)

Chabrier, G., Baraffe, I., Allard, F., \& Hauschildt, P. 2000, ApJ, 542, 464

Chapman, N. L., Lai, S.-P., Mundy, L. G., et al. 2007, ApJ, 667, 288

Chevalier, R. A. 1977, ARA\&A, 15, 175

Cieza, L., Padgett, F. L., Stapelfeldt, K. R., et al. 2007, ApJ, 667, 308

Comerón, F. 2004, in Organizations and Strategies in Astronomy, 5, ed. A. Heck (Kluwer Acad. Publ.)

Comerón, F. 2008, in Handbook of Star Forming Regions, Vol. II, ed. B. Reipurth, ASP Monograph Publ., 5, 295

Comerón, F., Fernández, M., Baraffe, I., et al. 2006, A\&A, 406, 1001

Dahn, C. C., Harris, H. C., Vrba, F. J., et al. 2002, AJ, 124, 1170

de Zeeuw, P. T., Hoogerwerf, R., de Bruijne, J. H .J., Brown, A. G. A., \& Blaauw, A. 1999, AJ, 117, 354

Dobashi, K., Uehara, H., Kandori, R., et al. 2005, PASJ, 57, S1

Evans, N. J., II, Allen, L. E., Blake, G. A., et al. 2003, PASP, 115, 965

Evans, N. J., et al. 2009, ApJS, in press

Fernández, M., \& Comerón, F., A\&A, 2005, 440, 1119

Fluks, M. A. 1998, Ph.D. Thesis, Univ. of Amsterdam

Fukugita, M., Ichikawa, T., Gunn, J. E., et al. 1996, AJ, 111, 1748

Gondoin, P. 2006, A\&A, 454, 595

Graham, J. A., \& Heyer, M. H. 1988, PASP, 100, 1529
Guillout, P., Sterzik, M. F., Schmitt, J. H. M. M., Motch, C., \& Neuhäuser, R. 1998, A\&A, 337, 113

Hauschildt, P. H., Allard, F., \& Baron, E. 1999, ApJ, 512, 377

Haisch, K. E., Lada, E. A., \& Lada, C. J. 2001, ApJ, 553, L153

Herbig, G. H. 1998, ApJ, 497, 736

Hughes, J. H., Hartigan, P., Krautter, J., \& Kelemen, J. 1994, AJ, 108, 1071

Jayawardhana, R., \& Ivanov, V. D. 2006, ApJ, 647, L167

Jayawardhana, R., Ardila, D. R., Stelzer, B., \& Haisch, K. E., 2003, AJ, 126, 1515

Johnstone, D., Hollenbach, D., \& Bally, J. 1998, ApJ, 499, 758

Kainulainen, J., Lehtinen, K., \& Harju, J. 2006, A\&A, 447, 597

Kenyon, S. J., \& Hartmann, L. 1995, ApJS, 101, 117

Kirkpatrick, J. D. 2005, ARA\&A, 43, 195

Koch, A., Grebel, E. K., Odenkirchen, M., \& Caldwell, J. A. R. 2003, The Messenger, 115, 37

Krautter, J., Wichmann, R., Schmitt, J. H. M. M., et al. 1997, A\&AS, 123, 329

Landolt, A. U. 1992, AJ, 104, 340

Lawson, W. A., Lyo, A.-R., \& Muzerolle, J. 2004, MNRAS, 351, L39

Lee, H. T., Chen, W. P., Zhi, W. Z., \& Jing, Y. H. 2005, ApJ, 624, 808

Li, J. Z., \& Rector, T. A. 2007, NewA, 12, 441

López Martí, B., Eislöffel, J., \& Mundt, R. 2005, A\&A, 440, 139

Luhman, K. L. 1999, ApJ, 525, 466

Luhman, K. L. 2007, ApJS, 173, 104

Luhman, K. L., \& Rieke, G. H. 1999, ApJ, 525, 440

Luhman, K. L., Stauffer, J. R., Muench, A. A. et al. 2003, ApJ, 593, 1093

Luhman, K. L., Allers, K. N., Jaffe, D. T., et al. 2007, ApJ, 659, 1629

Luhman, K. L., Allen, L. E., Allen, P. R., et al. 2008, ApJ, 675, 1375

Lyo, A.-R., Lawson, W. A., Mamajek, E. E., \& Feigelson, E. D. 2003, MNRAS 38,616

Merín, B., Augereau, J.-C., van Dishoeck, E. F. et al. 2007, ApJ, 661, 361

Merín, B., Jørgensen, J., Spezzi, L., et al. 2008, ApJ, submitted

Meyer, M. R., Calvet, N., \& Hillenbrand, L. A. 1997, ApJ, 114, 288

Nakajima, Y., Tamura, M., Oasa, Y., \& Nakajima, T. 2000, AJ, 119, 873

Oliveira, I., Merín, B., Pontoppidan, K. M., et al. 2009, ApJ, 691, 672

Preibisch, T., \& Zinnecker, H. 1999, AJ, 117, 2381

Reipurth, B. 2008, Handbook of Star Forming regions, ASP Monograph

Riaz, B., \& Gizis, J. E. 2008, ApJ, 681, 1584

Richling, S., \& Yorke, H. W. 1998, A\&A, 340, 508

Robberto, M., Song, J., Mora Carrillo, G., et al. 2004, ApJ, 606, 952

Robitaille, T. P., Whitney, B. A., Indebetouw, R., Wood, K., \& Denzmore, P. 2006, ApJS, 167, 256

Scholz, A., \& Jayawardhana, R. 2008, ApJ, 672, L49

Schwartz, R. D. 1977, ApJS, 35, 161

Skrutskie, M. F., Cutri, R. M., Stiening, R., et al. 2006, AJ, 131, 1163

Smith, J., Allyn, Tucker, D. L., Kent, S., et al. 2002, AJ, 123, 2121

Spezzi, L., Alcalá, J. M., Frasca, A., et al. 2007, A\&A, 470, 281

Stahler, S. W. 1983, ApJ, 274, 822

Steenman, H., Thé, P.-S. 1989, Ap\&SS, 159, 189

Sterzik, M. F., Alcalá, J. M., Neuhäuser, R., \& Schmitt, J. H. M. M. 1995, A\&A, 297, 418

Stetson, P. B. 1987, PASP, 99, 191

Thé, P. S. 1962, Contrib. Bosscha Obs., 15

Uchida, K. I., Calvet, N., Hartmann, L., et al. 2004, ApJS, 154, 439

Vrba, F. J., \& Rydgren, A. E. 1984, ApJ, 283, 123

Wainscoat, R. J., Cohen, M., Volk, K., Walker, H. J., \& Schwartz, D. E. 1992, ApJS, 83, 111

Weingartner, J. C., \& Draine, B. T. 2001, ApJ, 548, 296

Wichmann, R., Krautter, J., Covino, E., et al. 1997, A\&A, 320, 185 
F. Comerón et al.: A new population of cool stars and brown dwarfs in the Lupus clouds, Online Material $p 1$

Table 4. Derived physical parameters for known, non-saturated members of Lupus 1, 3, and 4.

\begin{tabular}{|c|c|c|c|c|c|c|c|c|c|c|}
\hline $\begin{array}{l}\text { Object } \\
(\mathrm{SSTc} 2 \mathrm{dJ})\end{array}$ & $\begin{array}{l}T_{\text {eff }} \\
(\mathrm{K})\end{array}$ & $\begin{array}{c}L \\
\left(L_{\odot}\right)\end{array}$ & $\begin{array}{c}A_{V} \\
(\mathrm{mag})\end{array}$ & $\begin{array}{c}M \\
\left(M_{\odot}\right)\end{array}$ & $\begin{array}{c}\text { Age } \\
\text { (Myr) }\end{array}$ & Other names & $\begin{array}{l}\text { Memb. } \\
\text { Ref. }\end{array}$ & Sp. type & $\begin{array}{l}\text { Sp. type } \\
\text { Ref. }\end{array}$ & Notes \\
\hline \multicolumn{11}{|c|}{ Lupus 1} \\
\hline $153928.3-344618$ & 3100 & 0.8 & 0.2 & 0.200 & 1.0 & Sz 66 & $\mathrm{~m} 1$ & M3 & s1 & \\
\hline $154457.9-342340$ & 3000 & 0.02 & 0.3 & 0.075 & 4.0 & $\# 19$ & $\mathrm{~m} 2$ & & & \\
\hline $154518.5-342125$ & 2700 & 0.09 & 1.6 & - & $<1.0$ & & $\mathrm{~m} 3$ & & & \\
\hline $154517.4-341829$ & 4600 & 0.6 & 4.6 & 1.0 & 22 & HW Lup & $\mathrm{m} 1$ & M1 & s1 & 20 \\
\hline $154508.9-341734$ & 4600 & 0.7 & 8.9 & 1.1 & 20 & & $\mathrm{~m} 3$ & & & \\
\hline $154240.3-341343$ & 3900 & 1.8 & 8.7 & 1.2 & 1.1 & & $\mathrm{~m} 3$ & & & \\
\hline $154214.6-341026$ & 4000 & 0.01 & 7.5 & - & - & & $\mathrm{m} 3$ & & & 1 \\
\hline $154140.8-334519$ & 2800 & 0.005 & 0.9 & 0.040 & 8.0 & $\# 18$ & $\mathrm{~m} 2$ & M8/M5.75 & $\mathrm{s} 2 / \mathrm{s} 3$ & \\
\hline $153803.1-331358$ & 3500 & 2.3 & 4.2 & - & $<1.0$ & & $\mathrm{~m} 3$ & & & \\
\hline \multicolumn{11}{|c|}{ Lupus 3} \\
\hline $161218.5-393418$ & 4000 & 0.024 & 3.6 & - & & & $\mathrm{m} 4$ & & & 1,34 \\
\hline $160917.1-392710$ & 2900 & 0.021 & 0.5 & 0.055 & 2.2 & Lup 710s & $\mathrm{m} 5$ & M5 & s7 & \\
\hline 160901.4-392512 & 4000 & 0.28 & 2.6 & 0.900 & 12.8 & & $\mathrm{~m} 3$ & & & 2 \\
\hline $160758.9-392435$ & 3000 & 0.058 & 0.9 & 0.130 & 2.3 & Lup 714 & $\mathrm{~m} 5$ & M5 & s7 & 29 \\
\hline $160837.3-392311$ & 10000 & 0.38 & 9.6 & - & - & Lup 706 & $\mathrm{~m} 5$ & L0 & s7 & 1,3 \\
\hline 161029.6-392215 & 3300 & 0.11 & 1.1 & 0.300 & 4.0 & & $\mathrm{~m} 3$ & & & \\
\hline $160737.7-392139$ & 3100 & 0.038 & 1.7 & 0.130 & 4.0 & Lup 713 s & $\mathrm{m} 5$ & M5.75 & s4 & \\
\hline $161012.2-392118$ & 3300 & 0.67 & 1.1 & 0.450 & 1.0 & Sz 121 & $\mathrm{~m} 1$ & M3 & s1 & \\
\hline $160754.1-392046$ & 3100 & 2.2 & 3.4 & - & $<1$ & & $\mathrm{~m} 3$ & & & \\
\hline $160942.6-391941$ & 5400 & 1.5 & 3.5 & - & - & Sz 116 & $\mathrm{~m} 1$ & M1.5 & s1 & $1,21,33$ \\
\hline $160754.7-391545$ & 4400 & 0.0069 & 6.4 & - & - & & $\mathrm{m} 6$ & & & $1,4,28,36$ \\
\hline $160853.2-391440$ & 3900 & 0.44 & 3.3 & 0.950 & 6.4 & & m6 & & & \\
\hline $160853.7-391437$ & 4000 & 0.064 & 8.4 & 0.600 & 159.9 & & $\mathrm{~m} 6$ & M5 & s4 & \\
\hline $160708.6-391407$ & 4400 & 0.078 & 7.1 & - & - & & $\mathrm{m} 3$ & & & $1,3,35,36$ \\
\hline 160934.1-391342 & 3500 & 10.3 & 6.4 & - & $<1$ & & $\mathrm{~m} 3$ & & & \\
\hline $160944.3-391330$ & 3700 & 0.47 & 1.5 & 0.750 & 3.2 & Sz 117 & $\mathrm{~m} 1$ & M2 & s1 & \\
\hline $160806.2-391223$ & 3900 & 5.1 & 8.1 & - & $<1$ & & $\mathrm{~m} 6$ & & & \\
\hline $160954.5-391204$ & 4400 & 0.17 & 4.4 & - & - & Lup 810 s & $\mathrm{m} 5$ & & & 1,7 \\
\hline $161126.0-391123$ & 3800 & 17.0 & 8.2 & - & $<1$ & & $\mathrm{~m} 3$ & & & 36 \\
\hline $160948.6-391117$ & 4800 & 4.3 & 8.9 & 1.400 & 11.3 & Sz 118 & $\mathrm{~m} 1$ & K6 & s1 & 36 \\
\hline $160703.9-391112$ & 3600 & 0.016 & 2.4 & - & - & & $\mathrm{m} 3$ & & & 1,8 \\
\hline $160710.1-391104$ & 3900 & 1.1 & 3.2 & 1.000 & 1.4 & $\mathrm{Sz} 90$ & $\mathrm{~m} 1$ & K7-M0 & s1 & \\
\hline $160848.2-390920$ & 2800 & 0.036 & 0.2 & 0.060 & 1.0 & Lup 617 & $\mathrm{~m} 5$ & M6 & s7 & \\
\hline $160906.2-390852$ & 3100 & 0.17 & 0.4 & 0.175 & 1.0 & Sz 115 & $\mathrm{~m} 1$ & M4 & s1 & \\
\hline $160812.6-390834$ & 4000 & 0.93 & 2.4 & 1.100 & 2.9 & Sz 96 & $\mathrm{~m} 1$ & M1.5 & s1 & 36 \\
\hline $161138.6-390828$ & 4400 & 0.37 & 6.1 & 0.950 & 25.2 & Lup 831s & $\mathrm{m} 5$ & & & \\
\hline 161016.4-390805 & 4800 & 0.62 & 1.8 & 0.950 & 35.6 & & $\mathrm{~m} 3$ & & & \\
\hline 160908.0-390726 & 4000 & 0.066 & 13.6 & - & - & & $\mathrm{m} 7$ & & & $22,32,36$ \\
\hline $160755.3-390718$ & 3900 & 9.9 & 12.3 & - & $<1$ & & $\mathrm{~m} 3$ & & & \\
\hline $161035.0-390655$ & 3600 & 2.7 & 7.1 & - & $<1$ & & $\mathrm{~m} 3$ & & & \\
\hline $161001.3-390645$ & 3200 & 0.22 & 4.4 & 0.250 & 1.1 & & $\mathrm{~m} 6$ & & & 9 \\
\hline $160839.8-390625$ & 4600 & 0.59 & 5.5 & 1.050 & 22.4 & Sz 106 & $\mathrm{~m} 1$ & $\mathrm{M} 2.5$ & s5 & 10,36 \\
\hline $160830.3-390611$ & 3000 & 0.17 & 0.3 & 0.150 & 1.3 & Sz 103 & $\mathrm{~m} 1$ & M4 & s1 & \\
\hline $160825.8-390601$ & 2900 & 0.24 & 0.5 & - & $<1$ & Sz 100 & $\mathrm{~m} 1$ & M5 & s5 & \\
\hline 160902.4-390549 & 4600 & 0.0079 & 4.4 & - & - & & $\mathrm{m} 7$ & & & $1,11,36$ \\
\hline $160824.0-390549$ & 7200 & 1.4 & 6.1 & - & - & Sz 99 & $\mathrm{~m} 1$ & M3.5 & s1 & 1 \\
\hline $160830.8-390549$ & 3000 & 0.13 & 0.4 & 0.150 & 1.3 & Sz 104 & $\mathrm{~m} 1$ & M5 & s1 & \\
\hline $160849.4-390539$ & 4400 & 1.0 & 6.0 & 1.400 & 5.7 & Par-Lup3-3 & $\mathrm{m} 8$ & M4.5 & s5 & 27 \\
\hline $160828.4-390532$ & 2900 & 0.39 & 0.2 & - & $<1$ & Sz 101 & $\mathrm{~m} 1$ & M4 & s1 & \\
\hline
\end{tabular}


F. Comerón et al.: A new population of cool stars and brown dwarfs in the Lupus clouds, Online Material p 2

Table 4. continued.

\begin{tabular}{|c|c|c|c|c|c|c|c|c|c|c|}
\hline $\begin{array}{l}\text { Object } \\
(\text { SSTc2dJ) }\end{array}$ & $\begin{array}{l}T_{\text {eff }} \\
(\mathrm{K}) \\
\end{array}$ & $\begin{array}{c}L \\
\left(L_{\odot}\right) \\
\end{array}$ & $\begin{array}{c}A_{V} \\
(\mathrm{mag})\end{array}$ & $\begin{array}{c}M \\
\left(M_{\odot}\right) \\
\end{array}$ & $\begin{array}{c}\text { Age } \\
(\mathrm{Myr})\end{array}$ & Other names & $\begin{array}{l}\text { Memb. } \\
\text { Ref. }\end{array}$ & Sp. type & $\begin{array}{l}\text { Sp. type } \\
\text { Ref. }\end{array}$ & Notes \\
\hline $160851.4-390530$ & 4400 & 0.021 & 5.3 & - & - & Par-Lup3-4 & $\mathrm{m} 8$ & M5 & s5 & $1,3,5,36$ \\
\hline $160901.8-390513$ & 2900 & 0.42 & 0.7 & - & $<1$ & Sz 114 & $\mathrm{~m} 1$ & M5.5 & s5 & \\
\hline $160723.4-390510$ & 4800 & 0.0098 & 3.2 & - & - & Lup 654 & $\mathrm{~m} 5$ & $\mathrm{~L} 1$ & s7 & 1 \\
\hline 160847.5-390509 & 4600 & 0.0086 & 5.7 & - & - & & m6 & & & 1 \\
\hline $160858.9-390446$ & 4400 & 0.0056 & 5.9 & - & - & & $\mathrm{m} 7$ & & & 1,22 \\
\hline $160749.6-390429$ & 3400 & 0.15 & 0.2 & 0.300 & 2.5 & Sz 94 & $\mathrm{~m} 1$ & M4 & s1 & \\
\hline $160828.2-390425$ & 4400 & 0.0097 & 9.5 & - & - & & $\mathrm{m} 7$ & & & $1,22,30,36$ \\
\hline $160821.8-390422$ & 3200 & 0.19 & 0.3 & 0.250 & 1.3 & Sz 97 & $\mathrm{~m} 1$ & M3 & s1 & \\
\hline $160848.2-390419$ & 2800 & 0.14 & 0.2 & - & $<1$ & Sz 109 & $\mathrm{~m} 1$ & M6.5 & s5 & \\
\hline $160853.7-390410$ & 4400 & 0.0047 & 3.2 & - & - & & $\mathrm{m} 7$ & & & $1,14,31$ \\
\hline $160835.8-390348$ & 3100 & 0.18 & 0.4 & 0.175 & 1.0 & Par-Lup3-2 & $\mathrm{m} 8$ & M6 & s5 & \\
\hline $160711.6-390348$ & 5400 & 1.2 & 4.3 & - & - & Sz 91 & $\mathrm{~m} 1$ & M0.5 & s1 & 24 \\
\hline $160908.5-390343$ & 3000 & 0.071 & 0.3 & 0.130 & 1.8 & Lup 608s & m5 & M5 & s7 & \\
\hline $160851.6-390318$ & 4400 & 0.5 & 1.6 & 1.050 & 20.0 & Sz 110 & $\mathrm{~m} 1$ & M4.5 & s5 & 25 \\
\hline $160829.7-390311$ & 4400 & 0.020 & 1.0 & - & - & Sz 102 & $\mathrm{~m} 1$ & K0: & s1 & $1,3,19,36$ \\
\hline 160816.0-390304 & 2800 & 0.080 & 2.0 & - & $<1$ & Par-Lup3-1 & $\mathrm{m} 8$ & M7.5 & s5 & \\
\hline $160800.2-390300$ & 2900 & 0.085 & 0.7 & 0.100 & 1.3 & Lup 604s & m5 & M5.25 & s4 & \\
\hline $160855.5-390234$ & 3000 & 0.24 & 0.4 & - & $<1$ & Sz 112 & $\mathrm{~m} 1$ & M6 & s5 & \\
\hline $161027.4-390230$ & 3700 & 0.0048 & 4.5 & - & - & & $\mathrm{m} 3$ & & & 1 \\
\hline $160857.8-390223$ & 3100 & 0.079 & 1.4 & 0.110 & 1.1 & Sz 113 & $\mathrm{~m} 1$ & M6 & s5 & \\
\hline $160841.8-390137$ & 3000 & 0.18 & 0.4 & 0.150 & 1.3 & Sz 107 & $\mathrm{~m} 1$ & M5.5 & s1 & \\
\hline $160956.3-385952$ & 2800 & 0.035 & 0.5 & 0.060 & 1.0 & Lup 818s & m5 & M6 & s7 & \\
\hline 160957.1-385948 & 3100 & 0.41 & 0.3 & - & $<1$ & Sz 119 & $\mathrm{~m} 1$ & M4 & s1 & \\
\hline $160752.3-385806$ & 3400 & 0.26 & 0.7 & 0.350 & 1.6 & Sz 95 & $\mathrm{~m} 1$ & M1.5 & s1 & \\
\hline $160815.0-385715$ & 4000 & 0.019 & 4.1 & - & - & & m6 & M4.75 & s4 & 1 \\
\hline $160859.5-385628$ & 2600 & 0.017 & 1.5 & - & $<1$ & & m6 & M8 & s4 & \\
\hline $161045.4-385455$ & 4400 & 0.030 & 2.7 & - & - & & $\mathrm{m} 3$ & & & 1 \\
\hline 161000.1-385401 & 2400 & 2.1 & 4.0 & - & - & & $\mathrm{m} 3$ & & & 15 \\
\hline $161051.6-385314$ & 3900 & 0.40 & 2.1 & 0.900 & 6.4 & Sz 123 & $\mathrm{~m} 1$ & M3 & s1 & \\
\hline $160714.0-385238$ & 2700 & 0.011 & 0.3 & 0.040 & 3.2 & Lup 605 & m5 & M6.5 & s7 & \\
\hline 160803.0-385229 & 3300 & 1.92 & 2.9 & - & $<1$ & & $\mathrm{~m} 3$ & & & \\
\hline $161151.2-385104$ & 3000 & 0.029 & 0.4 & 0.070 & 2.5 & Lup 802 s & m5 & M4 & s2 & \\
\hline $160949.9-384903$ & 2900 & 0.0041 & 0.8 & 0.050 & 12.6 & Lup 650 & m5 & M4 & s7 & \\
\hline $160855.3-384848$ & 2900 & 0.037 & 0.7 & 0.072 & 1.0 & & m6 & & & \\
\hline $161013.1-384617$ & 3500 & 0.0050 & 1.5 & - & - & & $\mathrm{m} 3$ & & & $1,16,36$ \\
\hline 161219.6-383742 & 3400 & 5.7 & 4.8 & - & $<1$ & & $\mathrm{~m} 3$ & & & \\
\hline $160927.0-383628$ & 5200 & 0.37 & 5.2 & - & - & & $\mathrm{m} 4$ & & & 1,3 \\
\hline 161018.6-383613 & 2900 & 0.045 & 0.2 & 0.080 & 1.1 & & $\mathrm{~m} 3$ & & & \\
\hline $161019.8-383607$ & 2700 & 0.024 & 0.2 & 0.040 & 1.1 & & $\mathrm{~m} 3$ & & & \\
\hline 161144.9-383245 & 2800 & 0.057 & 0.5 & 0.060 & 1.0 & & $\mathrm{~m} 3$ & & & \\
\hline $161211.2-383220$ & 4400 & 1.0 & 7.0 & 1.400 & 5.7 & & $\mathrm{~m} 3$ & & & 36 \\
\hline $161159.8-382339$ & 3100 & 0.084 & 0.9 & 0.110 & 1.0 & SST-Lup3-1 & $\mathrm{m} 9$ & M5.75 & s6 & \\
\hline $161148.7-381758$ & 4600 & 0.045 & 1.7 & - & - & & $\mathrm{m} 3$ & & & 1,17 \\
\hline $161243.8-381503$ & 4400 & 0.60 & 1.0 & 1.150 & 12.7 & & $\mathrm{~m} 3$ & & & \\
\hline $161131.9-381110$ & 4400 & 0.31 & 4.5 & 0.850 & 39.9 & & $\mathrm{~m} 3$ & & & \\
\hline $161204.5-380959$ & 4400 & 0.0056 & 3.6 & - & - & & $\mathrm{m} 3$ & & & 1,3 \\
\hline
\end{tabular}


F. Comerón et al.: A new population of cool stars and brown dwarfs in the Lupus clouds, Online Material p 3

Table 4. continued.

\begin{tabular}{|c|c|c|c|c|c|c|c|c|c|c|}
\hline $\begin{array}{l}\text { Object } \\
(\mathrm{SSTc} 2 \mathrm{dJ})\end{array}$ & $\begin{array}{l}T_{\text {eff }} \\
(\mathrm{K})\end{array}$ & $\begin{array}{c} \\
\left(L_{\odot}\right)\end{array}$ & $\begin{array}{c}A_{V} \\
(\mathrm{mag})\end{array}$ & $\begin{array}{c}M \\
\left(M_{\odot}\right)\end{array}$ & $\begin{array}{c}\text { Age } \\
(\mathrm{Myr})\end{array}$ & Other names & $\begin{array}{l}\text { Memb. } \\
\text { Ref. }\end{array}$ & Sp. type & $\begin{array}{l}\text { Sp. type } \\
\text { Ref. }\end{array}$ & Notes \\
\hline $161344.1-373646$ & 3400 & 0.056 & 1.0 & 0.400 & $\begin{array}{l}20.1 \\
\text { Lupus } 4\end{array}$ & & $\mathrm{~m} 4$ & & & \\
\hline $160002.4-422216$ & 5000 & 0.42 & 4.6 & - & - & & $\mathrm{m} 3$ & & & 1 \\
\hline $160000.6-422158$ & 3400 & 0.080 & 0.8 & 0.350 & 8.9 & & $\mathrm{~m} 3$ & & & \\
\hline $160129.7-420804$ & 4000 & 0.65 & 4.8 & 1.100 & 5.7 & & $\mathrm{~m} 3$ & & & \\
\hline $160026.1-415356$ & 3000 & 0.065 & 0.6 & 0.130 & 2.0 & & $\mathrm{~m} 3$ & & & \\
\hline $160229.9-415111$ & 3400 & 0.32 & 5.3 & 0.350 & 1.1 & & $\mathrm{~m} 3$ & & & \\
\hline $160031.1-414337$ & 3900 & 0.26 & 1.7 & 0.900 & 17.9 & Sz 130 & $\mathrm{~m} 1$ & M1.5 & s1 & \\
\hline $160329.4-414003$ & 4400 & 0.36 & 5.8 & 0.950 & 25.2 & Sz 133 & $\mathrm{~m} 1$ & $\mathrm{~K} 2$ & s1 & 26,36 \\
\hline $160111.6-413730$ & 3600 & 0.53 & 3.6 & 0.700 & 2.2 & & $\mathrm{~m} 3$ & & & \\
\hline $160143.3-413606$ & 3500 & 0.66 & 3.0 & 0.620 & 1.1 & & $\mathrm{~m} 3$ & & & \\
\hline $160049.4-413004$ & 4000 & 0.15 & 1.9 & 0.750 & 39.8 & Sz 131 & $\mathrm{~m} 1$ & M2 & s1 & 36 \\
\hline
\end{tabular}

\section{Membership references:}

m1: Schwartz (1977); m2: Allers et al. (2006); m3: Merín et al. (2008); m4: Chapman et al. (2007); m5: López Martí et al. (2005); m6: Allen et al. (2007); m7: Nakajima et al. (2000); m8: Comerón et al. (2003); m9: Merín et al. (2007).

Spectral type references:

s1: Hughes et al. (1994); s2: Jayawardhana \& Ivanov (2006); s3: Luhman et al. (2007); s4: Allen et al. (2007); s5: Comerón et al. (2003); s6: Merín et al. (2007); s7: López Martí et al. (2005)

Notes:

1: Apparently below the main sequence

2: Merín et al. (2008) fit a spectral type corresponding to a cooler temperature, with no extinction, and derive a lower luminosity that yields an unusually high disk-to-star luminosity ratio, $L_{\mathrm{disk}} / L_{*}$ ratio. Our results, involving moderate extinction, imply $L_{\mathrm{disk}} / L_{*}=0.36$, closer to typical values among the Lupus 3 population.

3: Unreliable fit probably due to veiling. See Table 6.

4: Important K excess in addition to veiling. No fit in Merín et al. (2008).

5: Well known to apparently lie below the main sequence despite its M 5 spectral type. Variability reported by (Comerón et al. 2003).

7: Fit as M5 in Merín et al. (2008); close companion at 2"4, probably contaminating the photometry confusion.

8: No fit in Merín et al. (2008).

9: Fit much later than in Merín et al. (2008)

10: Highly variable.

11: Candidate member in Nakajima et al. (2000). Variability suspected. A comparison with 2MASS photometry shows it to be fainter in J, H by 0.12 and 0.16 mag respectively, and brighter in Ks by $0.15 \mathrm{mag}$, in the observations of Nakajima et al. (2000).

14: Variability suspected. A comparison with 2MASS photometry shows it to be brighter in J by $0.26 \mathrm{mag}$, and slightly fainter in HKs by 0.07 mag. 15: Fitted by Merín et al. (2008) as A0 with very high extinction, which seems unlikely because it sits in a low extinction region. It may be a background red giant instead.

16: Strong K excess. No fit in Merín et al. (2008).

17: Relatively blue spectral energy distribution, the fit seems reasonable. Hints of elongation; perhaps having a disk close to edge-on orientation.

19: $\mathrm{Sz} 102$ is a peculiar object with strong emission lines, veiling and underluminous.

20: Very strong $\mathrm{H} \alpha$ emission reported by Hughes et al. (1994), possibly veiled.

21: Our measured $I_{\mathrm{C}}$ is fainter than that in Hughes et al. (1994), whereas the 2MASS $J H K_{S}$ magnitudes are brighter than those listed in that work by $0.4 \mathrm{mag}$. The source thus seems to be highly variable and our fit is likely to be affected by non-simultaneity of observations.

22: Selected as possible member by Nakajima et al. (2000) because of near-infrared excess.

24: Variability at the $\sim 0.2 \mathrm{mag}$ level in $J H K_{S}$ reported by Hughes et al. (1994). Our $I_{\mathrm{C}}$ measurement is very similar to that of Hughes et al. (1994). Probably variable.

25: Possibly variable: 2MASS $J$ magnitude brighter than in Hughes et al. (1994) by $\sim 0.2$ mag, but $H$ and $K_{S}$ almost identical. Our $I_{\mathrm{C}}$ measurement is $\sim 0.2$ mag brighter than in Hughes et al. (1994).

26: K2 in Hughes et al. (1994). The 2MASS $H K_{S}$ magnitudes are fainter than in Hughes et al. (1994) by $\sim 0.2$ mag, but $J$ is very similar. Our measurement in $I_{\mathrm{C}}$ is $0.5 \mathrm{mag}$ fainter than in Hughes et al. (1994). Probably variable, affecting our fit.

27: Moderately variable: the $R_{\mathrm{C}}$ and $I_{\mathrm{C}}$ in Comerón et al. (2003) are 0.17 mag fainter than in the new WFI measurements, and the 2MASS $H$ and $K_{S}$ magnitudes are $\sim 0.1$ mag brighter than the measurements in those bands given in Comerón et al. (2003).

28: Photometry in $R_{\mathrm{C}}$ contaminated by a much bluer companion at 1 " 7 , clearly detected in $R_{\mathrm{C}}$, much fainter in $I_{\mathrm{C}}$ and undetected in $z_{\mathrm{WFI}}$.

29: Extended in the NE-SW direction in the $R_{\mathrm{C}}$ image. Hints of elongation in $I_{\mathrm{C}}$, point source in $z_{\mathrm{WFI}}$. It might possess an outflow.

30: Very uncertain WFI photometry:marginal detection in $R_{\mathrm{C}}$ and $z_{\mathrm{WFI}}$, and very faint in $I_{\mathrm{C}}$.

31: Slightly fainter companion at $2 "$.

32: Undetected in $R_{\mathrm{C}}$ and faint in $I_{\mathrm{C}}$; uncertain fit at the WFI wavelengths.

33: Close companion at $1 " 5$ contaminating our photometry.

34: Clearly elongated in the NW-SE direction in $R_{\mathrm{C}}$, hints of elongation also in $I_{\mathrm{C}}$ and $z_{\mathrm{WFI}}$. It might be scattered light by a close-to-edge-on disk. 35: Slightly elongated in $R_{\mathrm{C}}$ and $I_{\mathrm{C}}$ in the NW-SE direction.

36: High residual fit (over $0.2 \mathrm{mag} \mathrm{rms}$ ). 
F. Comerón et al.: A new population of cool stars and brown dwarfs in the Lupus clouds, Online Material p 4

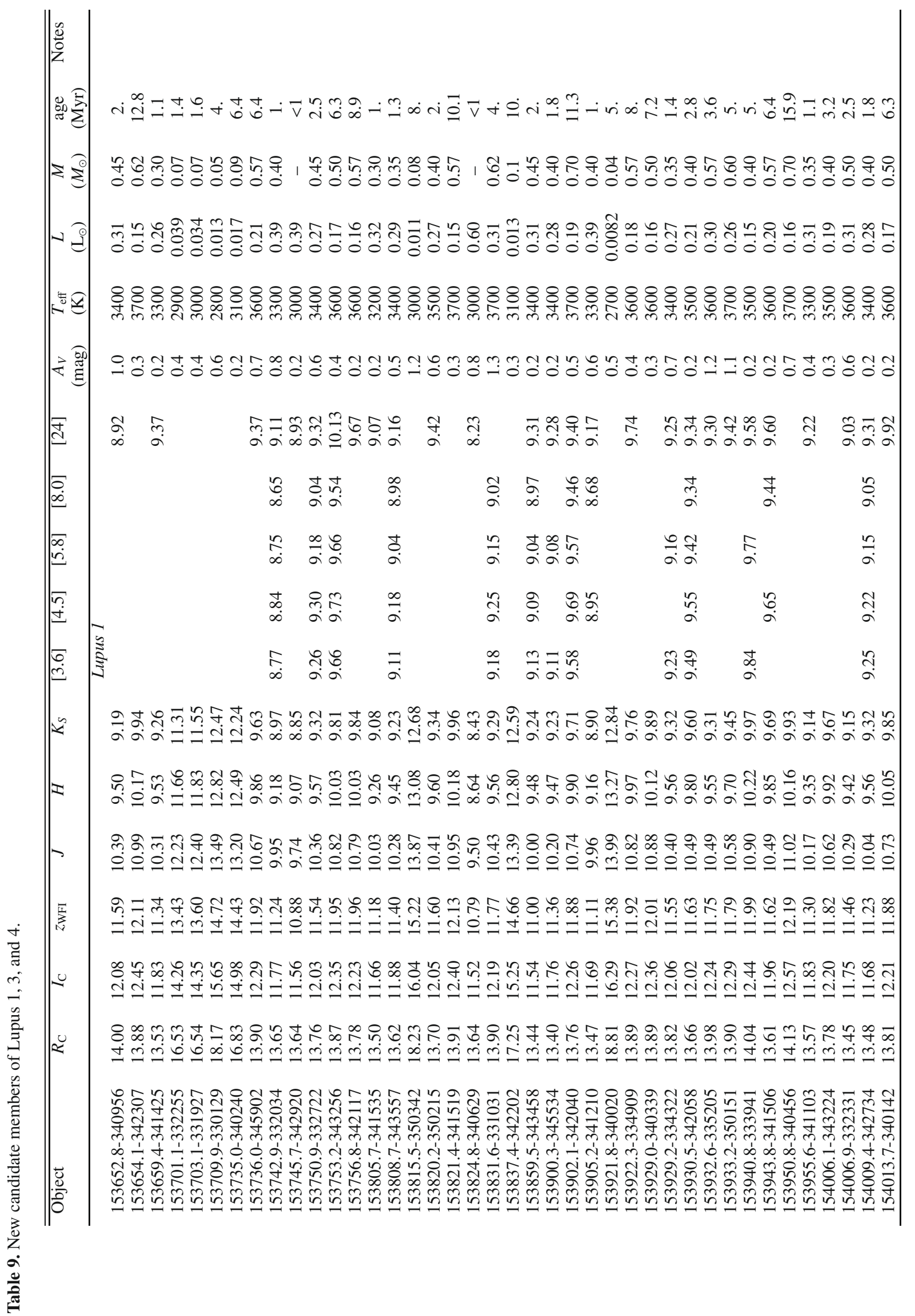


F. Comerón et al.: A new population of cool stars and brown dwarfs in the Lupus clouds, Online Material $p 5$

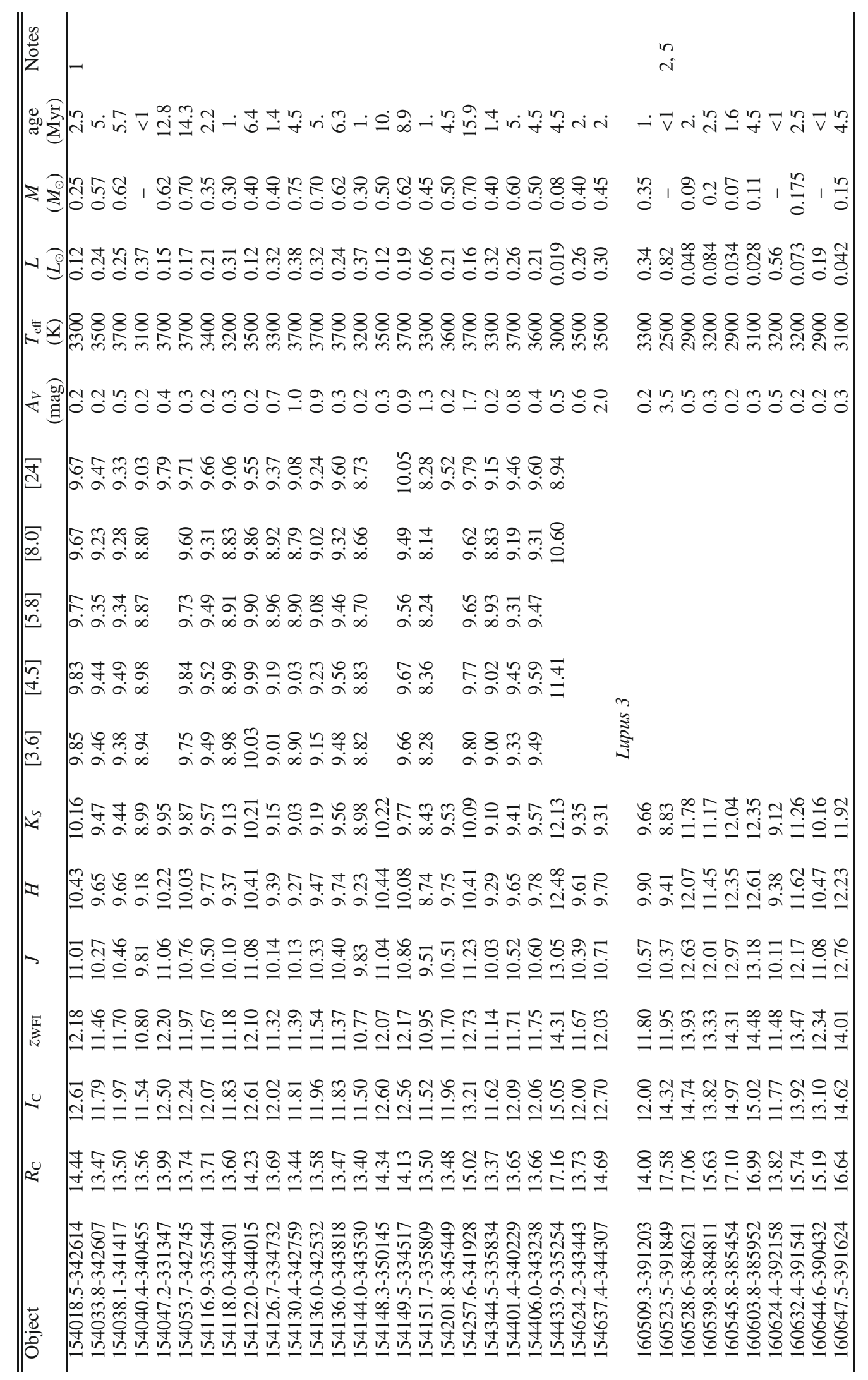


F. Comerón et al.: A new population of cool stars and brown dwarfs in the Lupus clouds, Online Material p 6

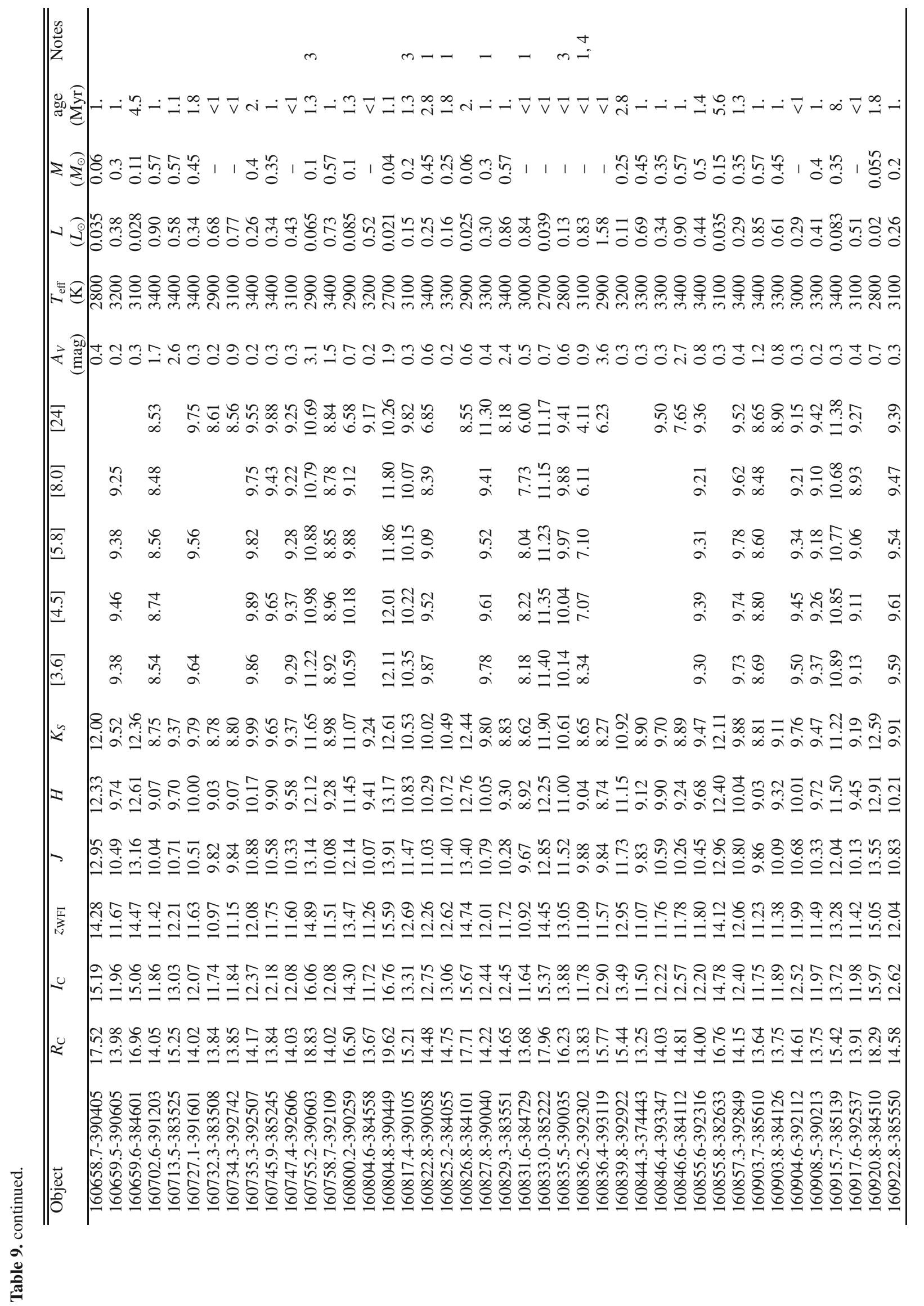


F. Comerón et al.: A new population of cool stars and brown dwarfs in the Lupus clouds, Online Material p 7

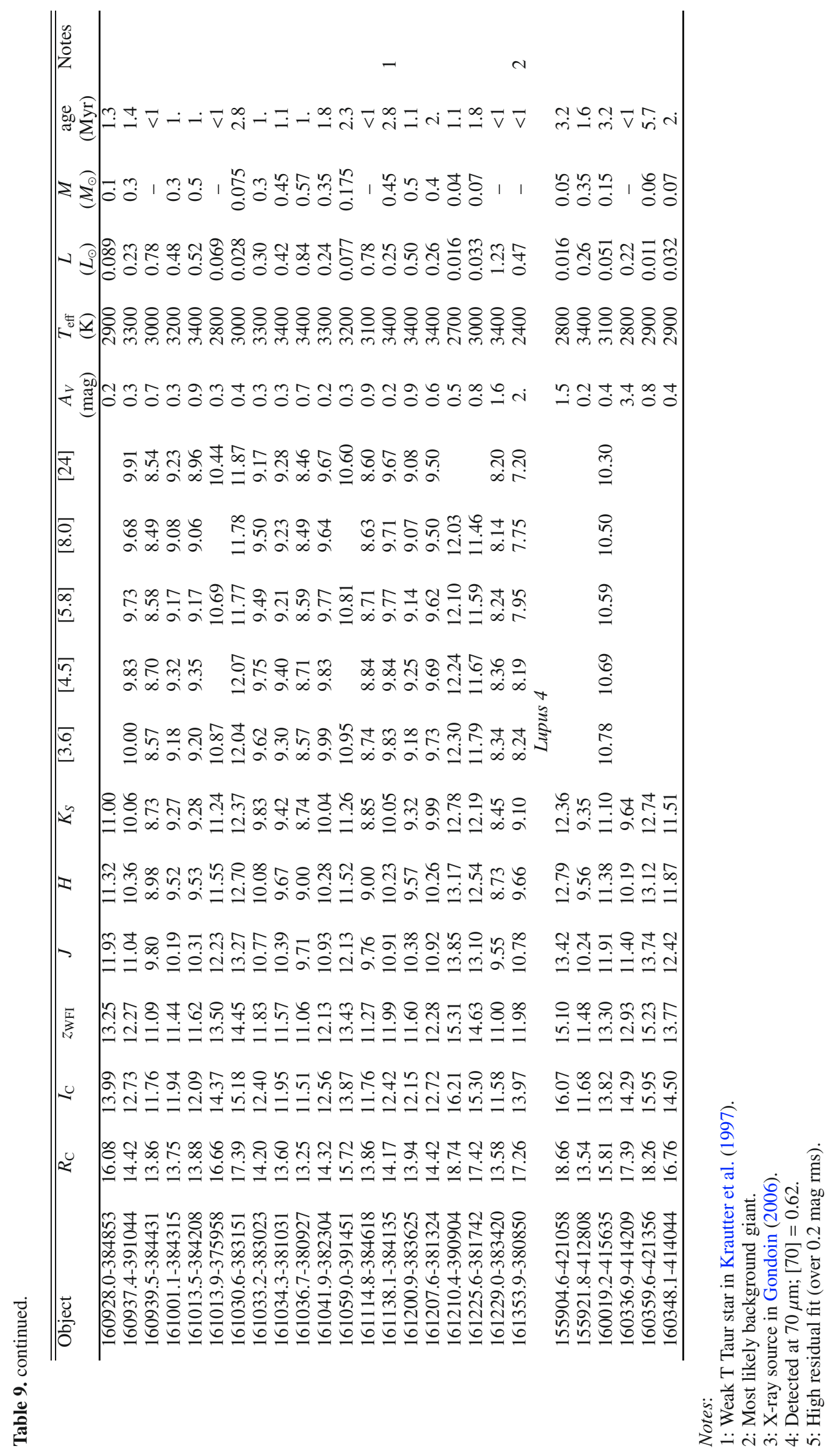

